Document downloaded from:

http://hdl.handle.net/10251/37056

This paper must be cited as:

González Albuixech, VF.; Giner Maravilla, E.; Tarancón Caro, JE.; Fuenmayor Fernández, FJ.; Gravouil, A. (2013). Domain integral formulation for 3-D curved and non-planar cracks with the extended finite element method. Computer Methods in Applied Mechanics and Engineering. 264:129-144. doi:10.1016/j.cma.2013.05.016.



The final publication is available at

http://dx.doi.org/10.1016/j.cma.2013.05.016

Copyright Elsevier 


\title{
Domain integral formulation for 3-D curved and non-planar cracks with the extended finite element method
}

\author{
Vicente F. González-Albuixech ${ }^{\mathrm{a}, 1}$, Eugenio Giner ${ }^{\mathrm{a}}$, \\ José E. Tarancón $^{\mathrm{a}}$, F. Javier Fuenmayor ${ }^{\mathrm{a}}$, Anthony Gravouil ${ }^{\mathrm{b}}$ \\ ${ }^{a}$ Centro de Investigación de Tecnología de Vehículos - CITV \\ Dpto. de Ingeniería Mecánica y de Materiales \\ Universitat Politècnica de València \\ Camino de Vera s/n, 46022 Valencia, Spain. \\ E-mail:vigonal@upvnet.upv.es \\ ${ }^{\mathrm{b}}$ Laboratoire de Mécanique des Contacts et des Structures (LaMCoS) \\ INSA-Lyon / UMR CNRS 5259 \\ 20 avenue Albert Einstein, F-69621 Villeurbanne, France
}

\begin{abstract}
The computation of stress intensity factors (SIF) in curved and non-planar cracks using domain integrals introduces some difficulties related to the use of curvilinear gradients. Several approaches exist in the literature that consider curvilinear corrections within a finite element framework, but these depend on each particular crack configuration and they are not general. In this work, we introduce the curvilinear gradient correction within the extended finite element method framework (XFEM), based only on the level set information used for the crack description and the local coordinate system definition. Our formulation depends only on the level sets coordinates and, therefore, an explicit analytical description of the crack is not needed. It is shown that this curvilinear correction improves the results and enables the study of generic cracks. In addition, we have introduced a simple error indicator for improving the SIF computed via the interaction integral, thanks to the better behaviour of the $J$-integral as it does not need auxiliary extraction fields.
\end{abstract}

Key words: XFEM, level sets, SIF, curved and non planar cracks, interaction integral, $J$-integral

PACS:

\footnotetext{
1 Laboratory for Nuclear Materials, Paul Scherrer Institut 5232 Villigen PSI, Switzerland

vicente.gonzalez@psi.ch
} 


\section{Introduction}

The accurate modeling of three-dimensional cracks in finite bodies remains a challenging problem in computational mechanics. The analysis of threedimensional fracture problems by the standard finite element method is difficult because of the need to construct a mesh which conforms to both the crack surface and the body geometry. The crack surface must be aligned with the element boundaries. Furthermore, for standard elements, the mesh must be designed so that is substantially refined around the crack front. The difficulties are further amplified for crack propagation studies, because the model must be remeshed at each growth step.

The extended finite element method (XFEM) is a technique that enables an efficient numerical modeling of crack problems $[1,2]$. The main idea of the method is to incorporate the crack geometry using enrichment functions within a finite element model, whose mesh does not need to conform to the crack boundaries. The enrichment is realized through the partition of unity concept. The method is useful for the approximation of solutions with pronounced non-smooth characteristics in small parts of the computational domain, for example discontinuities and singularities. In these cases, standard numerical methods, such as the FEM, often exhibit poor accuracy. However, the study of a generic 3-D problem using XFEM may still need the construction of a local refined mesh, although the element size should not be so refined as the adequate mesh for the standard finite element approach and the element topology does not need to match the crack geometry [3]. The XFEM and level set methods [4,5] also simplify the analysis and description of curved and/or non-planar cracks in three dimensions [6-8], as they provide the appropriate tools to build a local coordinate system natural to the crack geometry as shown in Fig. 1, where direction 1 is the normal direction to the crack front, contained in the crack plane, direction 2 is the normal direction to the crack surface and direction 3 is the tangential direction to the crack front.

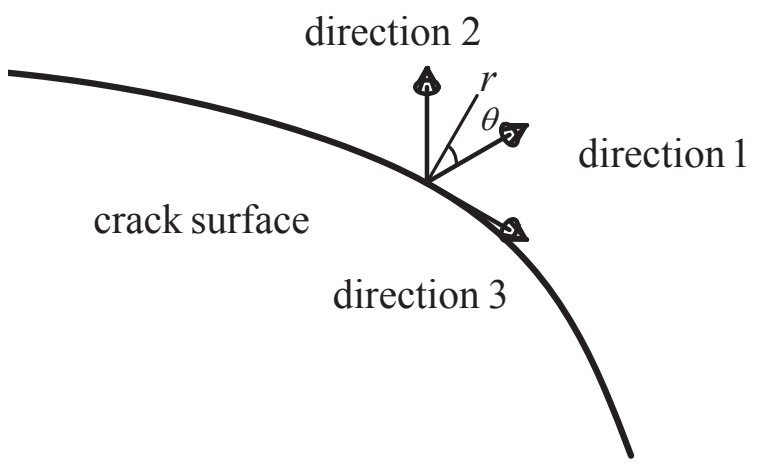

Fig. 1. Local reference coordinate system for a crack front.

On the other hand, not all the domain integrals are suitable for the study of 
curved or non planar cracks [9-11]. Domain integral formulations based on interaction integrals rely on the asymptotic crack field solution, which corresponds to a planar or straight case. The solution fields for curved cracks are not the same as the fields for a straight crack and, to the authors' knowledge, a generic expression is still not available. Consequently, the stress intensity factor extraction for curved crack needs a careful approach as shown in $[12,13]$ for 2-D problems. Gosz introduces corrections to the interaction integral in the study of $3-\mathrm{D}$ curved cracks using the finite element method $[9,10]$ achieving an improvement of the results. It includes the application of curvilinear differential geometry principles to the crack analysis. On the other hand, when a study is performed using the XFEM framework the crack is usually introduced using level sets $[1,2,6-8]$. Since the level sets introduce explicitly the crack surface information, an analogy to curvilinear analysis can be built. Our proposal is the introduction of a curvilinear gradient correction formulation for domain integrals in a XFEM framework using level sets. In this work, we also take advantage of the good behavior of the $J$-integral and its lack of dependency on auxiliary fields to introduce an easy way to improve the SIF calculations.

The paper is organized according to the following structure, where the main contributions are also noted:

- The paper starts with a review of XFEM and level set description for 3D cracks. We have proposed a level set orthogonal basis based on di erential geometry concepts.

- The domain integral formulation for the J-integral and the interaction integral are reviewed. The strong interaction integral assumption of considering straight planar cracks is remarked. We have introduced minor changes in the integral formulation and auxiliary field description to improve their performance in curved cracks studies.

- The domain integral computation in XFEM is addressed. We highlight that although in principle the XFEM is theoretically mesh independent, the use of a mesh related extraction domain for a non planar crack or curved crack analyses, introduces oscillations in the results.

- The derivatives of the curvilinear basis using only the level set information are developed using the differential curvilinear geometry theory. The curvilinear correction constitutes the main contribution of this work.

- We introduce a new error indicator that relies on the relative performance between J-integral and the interaction integral.

\section{XFEM for 3D cracks}

This section revisits the basis of the application of XFEM to 3-D crack studies for the sake of clarity and completeness. It covers a basic introduction to the 
XFEM enrichment and the level set crack description. It mainly introduces basic concepts extracted from more specific papers $[1,2,6-8]$, but it changes the traditional approach for the level set local basis to the viewpoint of a curved differential geometry framework.

\subsection{XFEM and enrichment for a $3 D$ case}

The XFEM is a numerical method that enables a local enrichment of the FE approximation spaces, realized through the partition of unity concept. The essential idea in XFEM is to use a displacement field approximation that can model any crack face discontinuity and the near-tip asymptotic crack field. As a consequence it is not necessary to modify the mesh to consider a specific crack; at most, moderate refinement must be introduced around the crack to achieve good accuracy. The method is based on the enrichment of the FE model with additional degrees of freedom (DOFs) that are associated with the nodes of the elements affected by the crack [1].

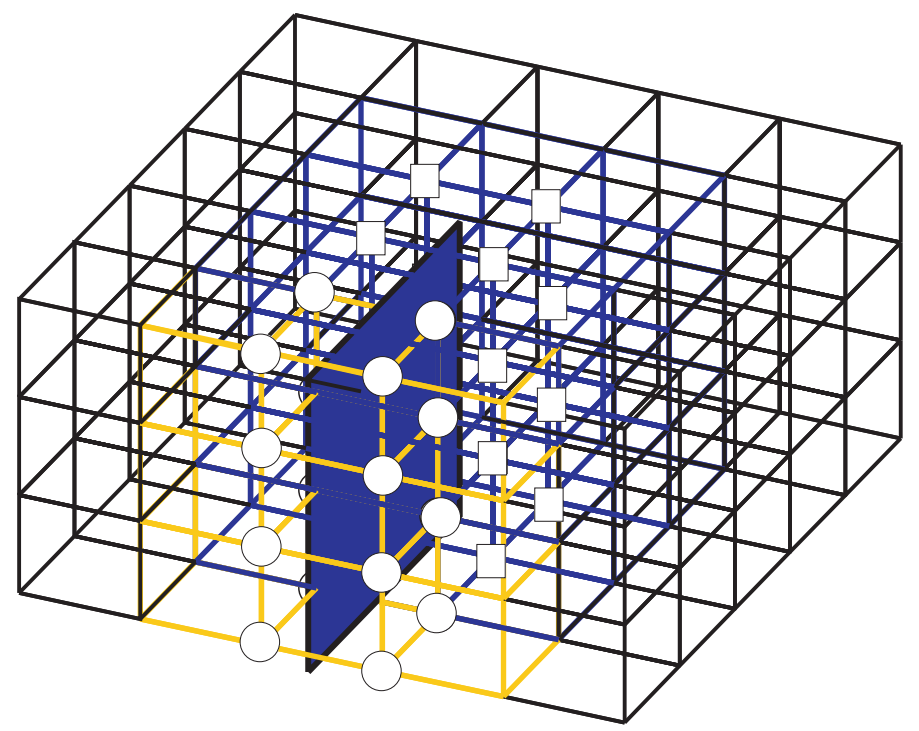

Fig. 2. Enriched nodes in the X-FEM. Circles: nodes with Generalized Heaviside function, 3 additional DOFs. Squares: nodes with crack tip enrichment functions, 12 additional DOFs.

Fig. 2 shows a portion of the mesh with linear hexahedron elements. Elements that contain at least one enriched node are known as enriched elements. Nodes with three additional DOFs (one for each coordinate direction) have shape functions that multiply the Generalized Heaviside function $H(\mathbf{x})$ (function of unit magnitude whose sign changes across the crack, $H(\mathbf{x})= \pm 1)$. Physically, this function introduces the discontinuity across the crack faces. Nodes with twelve additional DOFs are enriched in the three Cartesian directions with four crack tip functions $F_{\alpha}(\mathbf{x})[1]$ : 


$$
\left[F_{\alpha}(r, \theta), \alpha=1-4\right]=\left[\sqrt{r} \sin \frac{\theta}{2}, \sqrt{r} \cos \frac{\theta}{2}, \sqrt{r} \sin \frac{\theta}{2} \sin \theta, \sqrt{r} \cos \frac{\theta}{2} \sin \theta\right]
$$

where $r, \theta$ are local polar coordinates defined at the crack tip. We note that the span of the above functions can reproduce the asymptotic displacement fields in LEFM, which gives rise to the near-tip singular behavior in strains and stresses. It is well documented in the literature [1,14], and also verified through our studies that these functions significantly improve the accuracy of the SIF extraction.

The displacement approximation for crack modeling in the extended finite element method takes the form [1]:

$$
\mathbf{u}_{\mathrm{xfem}}(\mathbf{x})=\sum_{i \in \mathcal{I}} N_{i}(\mathbf{x}) \mathbf{u}_{i}+\sum_{i \in \mathcal{J}} N_{i}(\mathbf{x}) H(\mathbf{x}) \mathbf{a}_{i}+\sum_{i \in \mathcal{K}}\left[N_{i}(\mathbf{x}) \sum_{\alpha=1}^{4} F_{\alpha}(\mathbf{x}) \mathbf{b}_{i \alpha}\right]
$$

where $\mathcal{I}$ is the set of all nodes in the mesh, $N_{i}(\mathbf{x})$ are the nodal shape function and $\mathbf{u}_{i}$ are the standard DOFs of node $i\left(\mathbf{u}_{i}\right.$ represents the physical nodal displacement for non-enriched nodes only). The subsets $\mathcal{J}$ and $\mathcal{K}$ contain the nodes enriched with the Generalized Heaviside function $H(\mathbf{x})$ or the crack-tip functions $F_{\alpha}(\mathbf{x})$, respectively, and $\mathbf{a}_{i}, \mathbf{b}_{i \alpha}$ are the corresponding DOFs.

As in the standard FEM, it is necessary to perform numerical integration over the element domain to compute the element stiffness matrix. However, the elements that contain the crack include a displacement discontinuity due to the XFEM formulation. These elements are subdivided into subdomains, in which the crack is one of the subdomain boundaries, to carry out the numerical integrations. The algorithm presented in [15] is used to subdivide the elements totally cut by the crack. The integration over normal elements or over subdomains corresponding to the cut elements is performed using a normal Gauss-Legendre integration rule. The elements including the crack front are subdivided and integrated using a quasi polar rule introduced in [16].

\subsection{Level sets for crack geometry definition}

In addition to the numerical method, a description of the crack geometry is needed. The information of the crack is introduced through the use of distance functions based on level sets [6-8]. Two level sets are used for the crack description. They are denoted as $\phi$ and $\psi$ and can be observed in figure 3 . 
The $\phi$ level set is called the crack surface level set and its zero value corresponds to the crack surface. The $\psi$ level set, called front level set, determines the position relative to the crack front, which is located at the intersection of $\phi=0$ with $\psi=0$, and it is desirable that it be orthogonal to the level set $\phi$. In summary, the crack location is given by

$$
\begin{array}{ll}
\phi(\mathbf{x})=0 & \psi(\mathbf{x})<0 \longrightarrow \text { defines crack surface location } \\
\phi(\mathbf{x})=0 & \psi(\mathbf{x})=0 \longrightarrow \text { defines crack front location }
\end{array}
$$

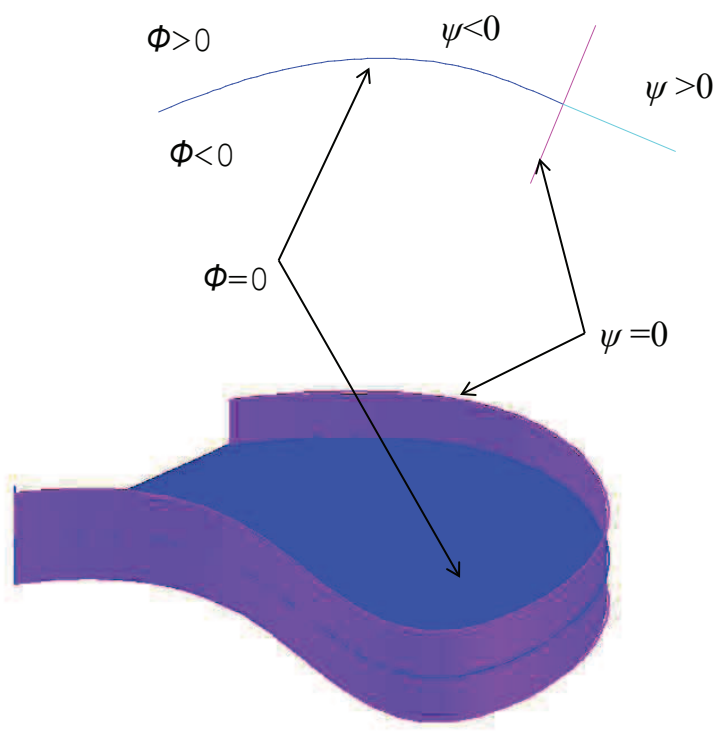

Fig. 3. Example of level sets for the general description of a crack.

The use of level sets for describing the crack has other advantages. First, the evaluation of level sets at these nodes of the elements allows to select which elements have to be enriched. This is accomplished by observing the change of sign of the distance functions in the element. Nodes that need to be enriched are the nodes which fulfill the conditions (4) where $I^{e l}$ is the set of nodes belonging to a given element $[6,8]$ :

$$
\begin{gathered}
\left\{\begin{array}{c}
\min _{i \in I^{e l}}\left(\operatorname{sign}\left(\phi_{i}(x, y)\right)\right) \max _{i \in I^{e l}}\left(\operatorname{sign}\left(\phi_{i}(x, y)\right)\right) \leq 0 \\
\max _{i \in I^{e l}}\left(\operatorname{sign}\left(\psi_{i}(x, y)\right)\right)<0
\end{array} \longrightarrow\right. \text { Heaviside enrichment } \\
\left\{\begin{array}{l}
\min _{i \in I^{e l}}\left(\operatorname{sign}\left(\phi_{i}(x, y)\right)\right) \max _{i \in I^{e l}}\left(\operatorname{sign}\left(\phi_{i}(x, y)\right)\right) \leq 0 \\
\min _{i \in I^{e l}}\left(\operatorname{sign}\left(\psi_{i}(x, y)\right)\right) \max _{i \in I^{e l}}\left(\operatorname{sign}\left(\psi_{i}(x, y)\right)\right) \leq 0
\end{array} \longrightarrow\right. \text { Crack front enrichment }
\end{gathered}
$$


Moreover the level set distance functions $\phi$ and $\psi$ can be used to build a curvilinear local basis associated with the crack geometry. The general local basis is defined using the normalized gradients of the level set functions (5) as in [6]. This, the level set local basis defines a natural system of coordinates. All tensors used are represented in this coordinate system.

$$
\begin{aligned}
\mathbf{e}_{1} & =\frac{\nabla \psi}{\|\nabla \psi\|} \\
\mathbf{e}_{2} & =\frac{\nabla \phi}{\|\nabla \phi\|} \\
\mathbf{e}_{3} & =\mathbf{e}_{1} \times \mathbf{e}_{2}
\end{aligned}
$$

Usually, it is assumed that the level sets are orthogonal in the sense that $\nabla \phi \nabla \psi=0$, but this is not true for the general case. It can be true locally but not globally, far away from the crack front. However, following an analogy to the surface differential geometry theory, it is possible to consider the plane defined by the basis vectors $\mathbf{e}_{1}$ and $\mathbf{e}_{3}$ as the tangent plane to the crack surface at the considered point. It allows to geometrically rebuild the other basis vector to obtain an orthogonal basis as $\mathbf{e}_{1}=\mathbf{e}_{3} \times \mathbf{e}_{2}$. This is a basis related to the Frenet trihedron and with the same meaning. The Frenet trihedron basis is clearly equivalent to the local basis, as can be inferred by comparison of Figs. 1 and 4. This basis description clearly remarks the existing relationships with differential geometry for curves and surfaces. This explicit formulation constitutes one of the contributions of our work. Our proposal for the basis definition and its exact relationship with level sets is:

$$
\begin{aligned}
\vec{N} & =\frac{\vec{T} \times \vec{B}}{\|\vec{T} \times \vec{B}\|}=\mathbf{e}_{1} \\
\vec{T} & =\frac{\nabla \psi \times \nabla \phi}{\|\nabla \psi \times \nabla \phi\|}=\mathbf{e}_{3} \\
\vec{B} & =\frac{\nabla \phi}{\|\nabla \phi\|}=\mathbf{e}_{2}
\end{aligned}
$$

In our implementation the values of the level sets are stored at the nodes of the finite element mesh. The usual element shape functions are employed for interpolating within the domain. The values of the vectors of the local basis, in the description of curved cracks, are computed at each element and averaged at the shared nodes with the neighboring elements $[7,8]$. To some extent, this maintains the curvature and allows to obtain an approximation to a continuous smooth local basis variation.

Another advantage that arises from the use of level sets and its associated local basis is the possibility of building polar coordinates at the crack front, which 


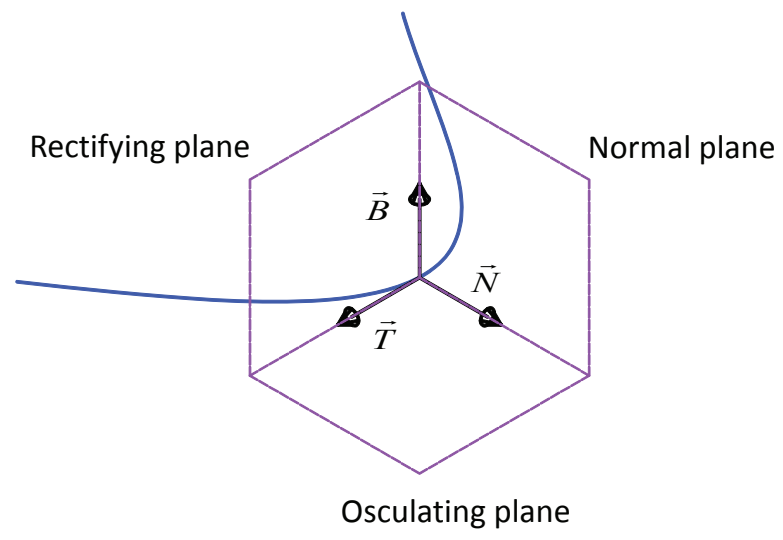

Fig. 4. Example of the Frenet trihedron for a curve.

provides directly $r$ and $\theta$ for the crack tip enrichment functions (1) as given in (7). On the other hand, the Generalized Heaviside enrichment matches the sign of the $\phi$ level set function.

$$
\begin{aligned}
& r=\sqrt{\psi^{2}+\phi^{2}} \\
& \theta=\tan ^{-1} \frac{\phi}{\psi}
\end{aligned}
$$

In this work, we will enforce the orthogonality of the level sets locally for all the numerical calculations, since it has been shown in [13] that it is necessary to guarantee convergence [3].

\section{Domain integral method for curved cracks}

We present a brief summary of the most used domain integrals. The formulation of the interaction integrals is revisited as it is usually based on the solution field of straight cracks. Some minor modifications are introduced in the interaction integral formulation to minimize the possible error associated with the use of straight crack fields. These improvements have been studied in [13] using convergence rates in 2D. The modifications do not depend on the dimensionality of the problem, and therefore the introduced formulation can be implemented in three dimensional cases.

\subsection{J-Integral}

The $J$-integral was introduced in [17] and it is one of the most powerful tools available for the extraction of the SIF, especially in its domain form [18]. An 
expression of the integral as applied to XFEM can be found in $[6,8]$. However it does not allow the extraction of the SIF for different modes in mixed mode situations. The domain form is given by:

$$
J=-\int_{V} \partial_{j} q_{i} P_{i j} \mathrm{~d} \Omega
$$

where $P_{i j}$ is the Eshelby's tensor [19], which is defined as

$$
P_{i j}=\frac{1}{2} \varepsilon_{k l} \sigma_{k l} \delta_{i j}-\sigma_{j k} \partial_{i} u_{k}
$$

where $u_{i}$ is the displacement field, $\sigma_{i j}$ is the stress field, $\delta_{i j}$ is the Kronecker's delta function and $q_{i}$ is a vector in direction $\mathbf{e}_{1}$ with a modulus proportional to a weight function $\alpha(\mathbf{x})$, which is zero on the contour of the extraction domain $A$ and one at the crack front point where $J$ is evaluated.

\subsection{Interaction Integral}

The interaction integral is used to extract the SIF under mixed-mode situations, $[6,8,11,20]$, enabling the computation of $K_{\mathrm{I}}$ and $K_{\mathrm{II}}$ in $2 \mathrm{D}$ problems. To achieve this goal, auxiliary fields are needed. The interaction integral is derived from the application of the $J$-integral to a problem where two stress fields are involved, resulting in the following decomposition:

$$
J^{(1+2)}=J^{(1)}+J^{(2)}+I
$$

The term $I$ corresponds to the interaction integral and includes the interaction between the two intervening fields. With no simplifications the general form of the interaction integral is

$$
\begin{aligned}
I= & -\int_{V}\left(\frac{1}{2}\left(\varepsilon_{k l}^{a u x} \sigma_{k l}+\varepsilon_{k l} \sigma_{k l}^{a u x}\right) \delta_{i j}-\sigma_{k j}^{a u x} \partial_{i} u_{k}-\sigma_{k j} \partial_{i} u_{k}^{a u x}\right) \partial_{j} q_{i} \mathrm{~d} \Omega \\
& -\int_{V}\left(\frac{1}{2}\left(\partial_{j} \varepsilon_{k l}^{a u x} \sigma_{k l}+\varepsilon_{k l}^{a u x} \partial_{j} \sigma_{k l}+\partial_{j} \varepsilon_{k l} \sigma_{k l}^{a u x}+\varepsilon_{k l} \partial_{j} \sigma_{k l}^{a u x}\right) \delta_{i j}\right) q_{i} \mathrm{~d} \Omega \\
& -\int_{V}\left(-\partial_{j} \sigma_{k j}^{a u x} \partial_{i} u_{k}-\sigma_{k j}^{a u x} \partial_{i j} u_{k}-\partial_{j} \sigma_{k j} \partial_{i} u_{k}^{a u x}-\sigma_{k j} \partial_{i j} u_{k}^{a u x}\right) q_{i} \mathrm{~d} \Omega
\end{aligned}
$$

The fields denoted with the superscript ${ }^{\text {aux }}$ are the auxiliary fields. Usually, the auxiliary fields are selected to be the straight crack fields, allowing the extraction of the different SIF modes. The fields $u_{i}, \sigma_{i j}, q_{i}$ and the Kronecker's delta $\delta_{i j}$ are defined in the same way as in the $J$-integral, being $\varepsilon_{i j}$ the strain 
field. For straight cracks the interaction integral can be simplified to achieve the well known expression $[6,8,11,20]$ :

$$
I=-\int_{V}\left(\sigma_{k l} \varepsilon_{k l}^{a u x} \delta_{i j}-\sigma_{k j}^{a u x} \partial_{i} u_{k}-\sigma_{k j} \partial_{i} u_{k}^{a u x}\right) \partial_{j} q_{i} \mathrm{~d} \Omega
$$

The use of the straight crack fields as auxiliary fields to evaluate a curved crack configuration, produces that (12) is not longer valid. The usual expression applied to evaluate the domain form of this integral for curved cracks $[1,6,8]$ is:

$$
\begin{aligned}
I= & -\int_{V}\left(\sigma_{k l} \varepsilon_{k l}^{a u x} \delta_{i j}-\sigma_{k j}^{a u x} \partial_{i} u_{k}-\sigma_{k j} \partial_{i} u_{k}^{a u x}\right) \partial_{j} q_{i} \mathrm{~d} \Omega \\
& -\int_{V}\left(\partial_{i} \sigma_{k l}^{a u x} \varepsilon_{k l} \delta_{i j}-\sigma_{k l} \partial_{l i} u_{k}^{a u x}-\partial_{l} \sigma_{k l}^{a u x} \partial_{i} u_{k}\right) q_{i} \mathrm{~d} \Omega
\end{aligned}
$$

The derivation of the expression (13) can be found, for example, in [11]. The generic treatment for applying the interaction integral to curved cracks is presented in $[9,10]$ and it is also analyzed for the $J$-integral in [23]. It is important to note that all the hypothesis assumed when deriving the above interaction integral expression, using the straight crack fields as auxiliary fields, should not be accepted directly for generic curved cracks [9-12]. However, they are all admitted in $[9,10]$.

The first problem arises from the fact that the inner equilibrium equation and the compatibility equations between strains-displacements of the auxiliary fields do not hold for a curved crack studied in curvilinear coordinates, that is

$$
\begin{aligned}
& \partial_{i} \sigma_{i j}^{a u x} \neq 0 \\
& \left(\partial_{l i} u_{j}^{a u x}-\partial_{l} \varepsilon_{i j}^{a u x}\right) \neq 0
\end{aligned}
$$

Therefore, the expression (12) cannot be used in curved cracks. In addition, the reciprocity relationship is also assumed in the derivation of (12) and (13). The reciprocity condition implies that the same constitutive tensor is used to relate the auxiliary strain field and the auxiliary stress field, i.e.

$$
\varepsilon_{i j}^{a u x} \sigma_{i j}=\varepsilon_{i j}^{a u x} C_{i j k l} \varepsilon_{k l}=C_{k l i j} \varepsilon_{i j}^{a u x} \varepsilon_{k l}=\sigma_{k l}^{a u x} \varepsilon_{k l}
$$

If this reciprocity condition is assumed, then the following relationship holds:

$$
\frac{1}{2}\left(\varepsilon_{i j}^{a u x} \sigma_{i j}+\varepsilon_{i j} \sigma_{i j}^{a u x}\right)=\varepsilon_{i j}^{a u x} \sigma_{i j}
$$


As a consequence, Eqs. (12) and (13) are obtained as a simplification of Eq. (11), as is usually done in the literature. The terms involved in (16) correspond to the interaction strain energy. This way, the auxiliary fields corresponding to a straight crack are enforced to be used with a curved coordinate system. This is the reason why the reciprocity relationship cannot be accepted and the simplifications (16) and (13) should not be used for curved crack problems.

The reciprocity relationship is also used in the derivation of the second integrand of (13) from Eq. (11), i.e.

$$
\partial_{i} \sigma_{k l}^{a u x} \varepsilon_{k l} \delta_{i j}-\sigma_{k l} \partial_{l i} u_{k}^{a u x}-\partial_{l} \sigma_{k l}^{a u x} \partial_{i} u_{k}
$$

is a simplification of

$$
\begin{gathered}
\frac{1}{2}\left(\partial_{j} \varepsilon_{k l}^{a u x} \sigma_{k l}+\varepsilon_{k l}^{a u x} \partial_{j} \sigma_{k l}+\partial_{j} \varepsilon_{k l} \sigma_{k l}^{a u x}+\varepsilon_{k l} \partial_{j} \sigma_{k l}^{a u x}\right) \delta_{i j} \\
-\partial_{j} \sigma_{k j}^{a u x} \partial_{i} u_{k}-\sigma_{k j}^{a u x} \partial_{i j} u_{k}-\partial_{j} \sigma_{k j} \partial_{i} u_{k}^{a u x}-\sigma_{k j} \partial_{i j} u_{k}^{a u x}
\end{gathered}
$$

If the reciprocity relationship is not applied in (18), the computation of derivatives of the numerical approximation near the crack faces are required, such as $\partial_{j} \sigma_{k l}$ and $\partial_{j} \varepsilon_{k l}$. The numerical computation of these derivatives introduces large numerical errors. However, we have verified that the integrand (18) has little influence on the final result. Hence, to avoid the introduction of further numerical errors, we will admit the reciprocity relationship only in the derivation of the simplified expression (17). Therefore, the interaction integral expression that we will use in this work is finally:

$$
\begin{aligned}
I= & -\int_{V}\left(\frac{1}{2}\left(\sigma_{k l} \varepsilon_{k l}^{a u x}+\sigma_{k l}^{a u x} \varepsilon_{k l}\right) \delta_{i j}-\sigma_{k j}^{a u x} \partial_{i} u_{k}-\sigma_{k j} \partial_{i} u_{k}^{a u x}\right) \partial_{j} q_{i} \mathrm{~d} \Omega \\
& -\int_{V}\left(\partial_{i} \sigma_{k l}^{a u x} \varepsilon_{k l} \delta_{i j}-\sigma_{k l} \partial_{l i} u_{k}^{a u x}-\partial_{l} \sigma_{k l}^{a u x} \partial_{i} u_{k}\right) q_{i} \mathrm{~d} \Omega
\end{aligned}
$$

The former interaction integral expression shows the best behavior, at least in 2-D situations, as tested in [13].

\subsubsection{Auxiliary fields}

Due to the lack of knowledge of a general expression for the auxiliary fields for curved cracks, the first terms of the Williams' asymptotic fields of the LEFM are used as auxiliary fields, using the definitions stated in (7) as $\theta$ and $r$. 
However, it is important to note that for general curved cracks, the conditions of compatibility, inner equilibrium and Hooke's law are not simultaneously verified for this selection of the auxiliary fields $[9,10,12]$. The auxiliary displacement field for a straight crack is the first term of the Williams' series expansion:

$$
\begin{aligned}
& u_{1}^{a u x}=\frac{1}{2 \mu} \sqrt{\frac{r}{2 \pi}}\left\{K_{\mathrm{I}}^{\text {aux }} \cos \frac{\theta}{2}(\kappa-\cos \theta)+K_{\mathrm{II}}^{\text {aux }} \sin \frac{\theta}{2}(\kappa+2+\cos \theta)\right\} \\
& u_{2}^{a u x}=\frac{1}{2 \mu} \sqrt{\frac{r}{2 \pi}}\left\{K_{\mathrm{I}}^{\text {aux }} \sin \frac{\theta}{2}(\kappa-\sin \theta)+K_{\mathrm{II}}^{\text {aux }} \cos \frac{\theta}{2}(\kappa-2+\cos \theta)\right\}
\end{aligned}
$$

where, assuming plane strain state for 3-D [24]

$$
\mu=\frac{E}{2(1+\nu)} \quad \kappa=3-4 \nu
$$

The auxiliary stress field is:

$$
\begin{aligned}
\sigma_{11}^{\text {aux }} & =\frac{K_{\mathrm{I}}^{\text {aux }}}{\sqrt{2 \pi r}}\left[1-\sin \frac{\theta}{2} \sin \frac{3 \theta}{2}\right] \cos \frac{\theta}{2}-\frac{K_{\mathrm{II}}^{\text {aux }}}{\sqrt{2 \pi r}}\left[2+\cos \frac{\theta}{2} \cos \frac{3 \theta}{2}\right] \sin \frac{\theta}{2} \\
\sigma_{22}^{\text {aux }} & =\frac{K_{\mathrm{I}}^{\text {aux }}}{\sqrt{2 \pi r}}\left[1+\sin \frac{\theta}{2} \sin \frac{3 \theta}{2}\right] \cos \frac{\theta}{2}+\frac{K_{\mathrm{II}}^{\text {aux }}}{\sqrt{2 \pi r}} \cos \frac{\theta}{2} \cos \frac{3 \theta}{2} \sin \frac{\theta}{2} \\
\sigma_{33}^{\text {aux }} & =\nu\left(\sigma_{11}+\sigma_{22}\right) \\
\sigma_{12}^{\text {aux }} & =\frac{K_{\mathrm{I}}^{\text {aux }}}{\sqrt{2 \pi r}} \cos \frac{\theta}{2} \cos \frac{3 \theta}{2} \sin \frac{\theta}{2}+\frac{K_{\mathrm{II}}^{\text {aux }}}{\sqrt{2 \pi r}}\left[1-\sin \frac{\theta}{2} \sin \frac{3 \theta}{2}\right] \cos \frac{\theta}{2} \\
\sigma_{13}^{\text {aux }} & =-\frac{K_{\mathrm{III}}^{\text {aux }}}{\sqrt{2 \pi r}} \sin \frac{\theta}{2} \\
\sigma_{23}^{\text {aux }} & =\frac{K_{\mathrm{III}}^{\text {aux }}}{\sqrt{2 \pi r}} \cos \frac{\theta}{2}
\end{aligned}
$$

The auxiliary field for the strains is also needed. Two possible choices arise to obtain the strain field. The first choice is based on the enforcement of the constitutive strain-stress relationship $[6,8]$ and the strain field is computed from the stress field by applying the Hooke's law:

$$
\varepsilon_{i j}^{a u x}=C_{i j k l}^{-1} \sigma_{k l}^{a u x}
$$

The second option is that the strain field can be obtained from the deriva- 
tives of the displacement field like in [9-11], enforcing the strain-displacement relationship:

$$
\varepsilon_{i j}^{a u x}=\left\{\nabla_{\text {sim }} u^{a u x}\right\}_{i j}
$$

In principle, there is no reason to assume that either choice is the best one. As neither expression verifies simultaneously the compatibility and inner equilibrium relationships for the auxiliary fields, an averaged strain field between the two options has also been considered in [13]. The objective is to verify an averaged condition between compatibility and inner equilibrium relationship. The averaged auxiliary strain field is given by:

$$
\varepsilon_{i j}^{a u x}=\frac{\left\{\nabla_{s i m} u^{a u x}\right\}_{i j}+C_{i j k l}^{-1} \sigma_{k l}^{a u x}}{2}
$$

This averaged field o ption o ption $g$ ives t he $b$ est $b$ ehavior $f$ or $2 \mathrm{D}$ situations in [13], and consequently has been accepted as the best choice for our formulation. This averaged auxiliary strain field formulation does not depend on the problem dimensionality and its use in three dimensions also constitutes a contribution of this work.

We remark that the auxiliary fields used assume that a plane strain state is present in the proximity of the crack front. However, this is not true in the vicinity of the intersection of crack fronts with free boundaries.

\subsection{SIF extraction}

The $J$ integral verifies

$$
J=\int_{C} \alpha G \mathrm{~d} c
$$

and the interaction integral verifies

$$
I=\int_{C} \alpha G^{a u x} \mathrm{~d} c
$$

where $C$ is the length of the crack front included in the extraction domain and $G$ and $G^{\text {aux }}$ are energy magnitudes that are related to the SIF. The pointwise value of $G$ verifies the next relationship with the local SIFs in 3-D problems 


$$
G=\frac{\left(1-\nu^{2}\right)}{E}\left\{K_{\mathrm{I}}^{2}+K_{\mathrm{II}}^{2}\right\}+\frac{1}{2 \mu} K_{\mathrm{III}}^{2}
$$

and similarly for $G^{\text {aux }}$

$$
G^{a u x}=\frac{2\left(1-\nu^{2}\right)}{E}\left\{K_{\mathrm{I}} K_{\mathrm{I}}^{\text {aux }}+K_{\mathrm{II}} K_{\mathrm{II}}^{\text {aux }}\right\}+\frac{1}{\mu} K_{\mathrm{III}} K_{\mathrm{III}}^{\text {aux }}
$$

If the SIFs are assumed as constants in the integration domain, assumption valid for small extraction domains, then,

$$
\frac{\left(1-\nu^{2}\right)}{E}\left\{K_{\mathrm{I}}^{2}+K_{\mathrm{II}}^{2}\right\}+\frac{1}{2 \mu} K_{\mathrm{III}}^{2}=\frac{J}{\operatorname{meas}(C)}
$$

where meas $(C)$ is the integration of the virtual crack extension $\alpha$ along the crack front length of the extraction domain

$$
\operatorname{meas}(C)=\int_{C} \alpha \mathrm{d} c
$$

The computation for the SIF for each mode using the interaction integral $I$ is given by the following equations

$$
\begin{aligned}
K_{\mathrm{I}} & =\frac{E}{2\left(1-\nu^{2}\right)} \frac{I^{(\mathrm{I})}}{\text { meas }(C)} \quad \text { with } \quad K_{\mathrm{I}}^{\text {aux }}=1, \quad K_{\mathrm{II}}^{\text {aux }}=K_{\mathrm{III}}^{\text {aux }}=0 \\
K_{\mathrm{II}} & =\frac{E}{2\left(1-\nu^{2}\right)} \frac{I^{(\mathrm{II})}}{\text { meas }(C)} \quad \text { with } \quad K_{\mathrm{II}}^{\text {aux }}=1, \quad K_{\mathrm{I}}^{\text {aux }}=K_{\mathrm{III}}^{\text {aux }}=0 \\
K_{\mathrm{III}} & =\mu \frac{I^{(\mathrm{III})}}{\text { meas }(C)} \quad \text { with } \quad K_{\mathrm{III}}^{\text {aux }}=1, \quad K_{\mathrm{I}}^{\text {aux }}=K_{\mathrm{II}}^{\text {aux }}=0
\end{aligned}
$$

$I^{(\mathrm{m})}$ indicates that the interaction integral is computed using an auxiliary field where only the corresponding mode $m=$ I, II or III is non zero, [6,8-11].

\section{Extraction Domain computation in XFEM}

The integrals (8), (11), (12) and (13) are expressed in domain formulation which uses the virtual velocity field $q_{i}[6,8]$. The equivalent contour formulation is not well suited for numerical computations in the finite element frame- 
work. As a consequence, the selection of the virtual velocity field needs to be addressed. The extraction domain is controlled through the use of the virtual velocity field and the numerical integration is performed within the extraction domain using integration points at the element level. The extraction domain computation at the mesh nodes, although widely used, introduces an integration domain that is mesh dependent. This mesh dependency produces that the domain is not convex and some oscillations exist in the results. The importance of this fact when using XFEM is remarked in this work.

The computation for a crack front point $\mathrm{P}$ is done using a change of coordinate system from a Cartesian coordinate system centered in $\mathrm{P}$ to the local coordinated system centered also in $\mathrm{P}$ with coordinates $\xi_{1}, \xi_{2}$, and $\xi_{3}$, respectively in directions $\mathbf{e}_{1}, \mathbf{e}_{2}$ and $\mathbf{e}_{3}$. The virtual velocity field must be tangent to the crack faces and is defined by the expression:

$$
\mathbf{q}=\alpha\left(\xi_{1}, \xi_{2}, \xi_{3}\right) \mathbf{e}_{1}
$$

Note that the domain extraction region and the weight of the integration on the extraction domain is controlled through the use of $\alpha$, which depends on the level set coordinates. The function $\alpha$ has to take unit value at the crack front and zero on the boundary of the extraction domain.

The integration volume is a tubular domain centered at the crack point $\mathrm{P}$, where the integral is computed, see figure 5 . The points $\mathrm{P}$ selected to perform the domain integrals are either the intersection of the crack with the element faces or nodes. The tubular domain is defined using the level set basis and coordinates. In the local coordinate system it corresponds to a cylinder, centered in $\mathrm{P}$.

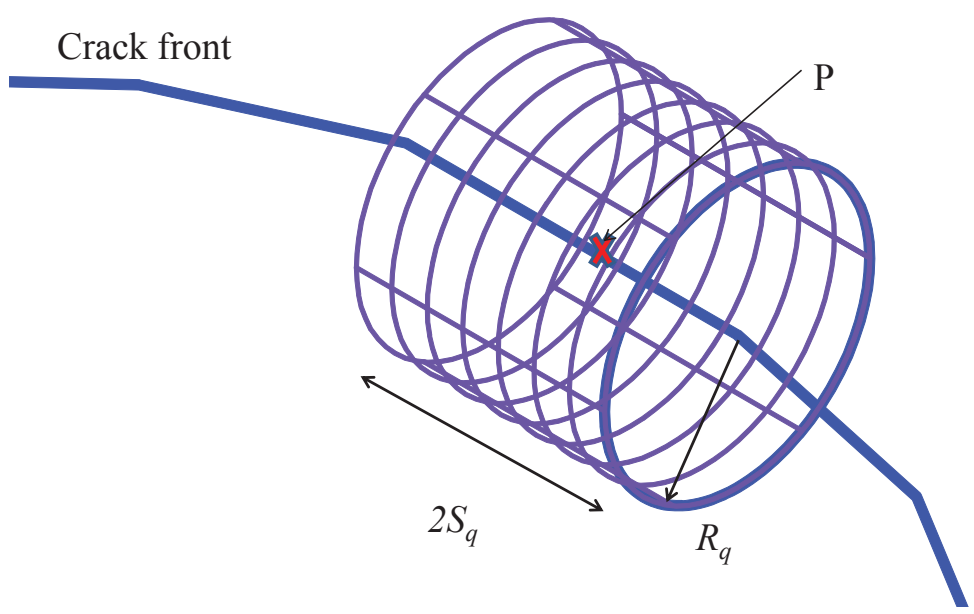

Fig. 5. Three dimensional extraction domain. 


$$
\alpha= \begin{cases}\left(1-\frac{\sqrt{\xi_{1}^{2}+\xi_{2}^{2}}}{R_{q}}\right)\left(1-\frac{\left|\xi_{3}\right|}{S_{q}}\right) & \text { if } \sqrt{\xi_{1}^{2}+\xi_{2}^{2}}<R_{q} \text { and }\left|\xi_{3}\right|<S_{q} \\ 0 & \text { if } \sqrt{\xi_{1}^{2}+\xi_{2}^{2}} \geq R_{q} \text { or }\left|\xi_{3}\right| \geq S_{q}\end{cases}
$$

In practice, the extraction domain size has influence on the convergence rate and on the computation of the SIF for cracks with generic curvature as shown in [13] for 2-D. Consequently the domain size has to be controlled. Moreover as the integration is performed at each integration point where the domain integral has to be computed, then the effective domain size suffers oscillations depending on the mesh topology as can be observed in figures 6 and 7 . These oscillations produce variations in the SIF computations and, as a consequence, the optimum convergence rate cannot be achieved, which is mainly due to the fact that the domain is not convex.
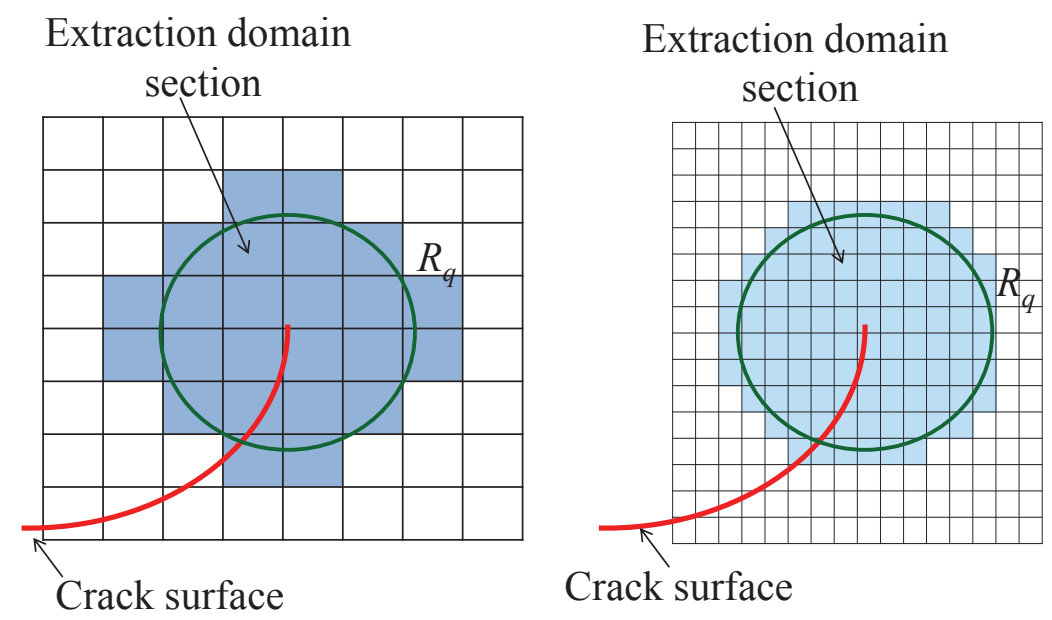

Fig. 6. Section of an extraction domain for a non-planar crack. 


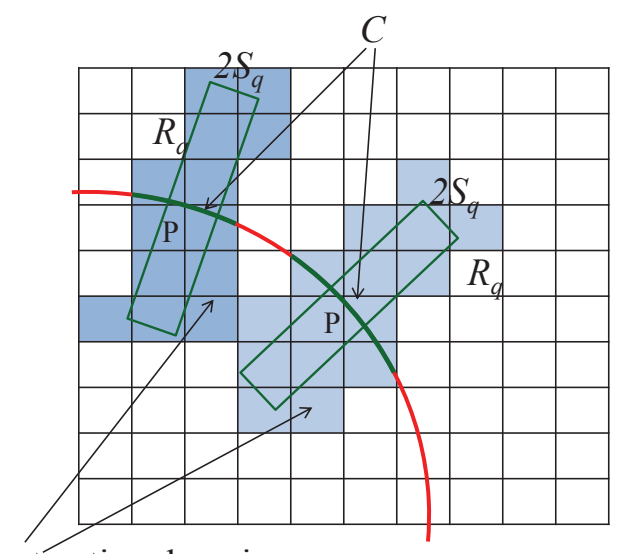

\section{Effective extraction domains Crack front} on the crack plane

Fig. 7. Extraction domains on the crack plane for a curved crack front.

\section{Derivatives on the curvilinear local basis}

The derivation in a local curvilinear coordinate system framework needs a change in the formulation and the introduction of new terms. In $[9,10]$ the curvilinear derivatives for the crack local basis in the finite element framework are introduced for the auxiliary field a nd t he $\mathrm{v}$ irtual v elocity fi eld. In this section we introduce our proposal for the derivatives in the XFEM framework, using the level set basis as local basis. In our approach the derivatives depend only on the level set definition and not explicitly on the problem to be solved, as in the FE expressions formulated by Gosz et al. [9,10]. The numerical derivation of these terms using the level set information in the di erential geometry framework is the main contribution of this work.

The gradient operator in curvilinear coordinates is defined as

$$
\partial_{i}=\mathbf{e}_{i} \frac{1}{h_{i}} \frac{\partial}{\partial \xi_{i}}
$$

where $h_{i}$ is the scale factor and $\xi_{i}$ is the respective curvilinear coordinate. On the other hand, the tensorial product of the gradient operator with a generic vectorial field, $\partial_{i} f_{j}$ is denoted as

$$
\partial_{i} f_{j}=\vec{\nabla} \otimes \vec{f}
$$

This equation is expressed in curvilinear coordinates as 


$$
\vec{\nabla} \otimes \vec{f}=\left(\mathbf{e}_{1} \frac{1}{h_{1}} \frac{\partial}{\partial \xi_{1}}+\mathbf{e}_{2} \frac{1}{h_{2}} \frac{\partial}{\partial \xi_{2}}+\mathbf{e}_{3} \frac{1}{h_{3}} \frac{\partial}{\partial \xi_{3}}\right) \otimes\left(\mathbf{e}_{1} f_{1}+\mathbf{e}_{2} f_{2}+\mathbf{e}_{3} f_{3}\right)
$$

Using matrix notation, the following expression is obtained.

$$
\begin{aligned}
& \vec{\nabla} \otimes \vec{f}=\left(\begin{array}{cccc}
\frac{1}{h_{1}} \frac{\partial f_{1}}{\partial \xi_{1}} & \frac{1}{h_{1}} \frac{\partial f_{2}}{\partial \xi_{1}} & \frac{1}{h_{1}} \frac{\partial f_{3}}{\partial \xi_{1}} \\
\frac{1}{h_{2}} \frac{\partial f_{1}}{\partial \xi_{2}} & \frac{1}{h_{2}} \frac{\partial f_{2}}{\partial \xi_{2}} & \frac{1}{h_{2}} \frac{\partial f_{3}}{\partial \xi_{2}} \\
\frac{1}{h_{3}} \frac{\partial f_{1}}{\partial \xi_{3}} & \frac{1}{h_{3}} \frac{\partial f_{2}}{\partial \xi_{3}} & \frac{1}{h_{3}} \frac{\partial f_{3}}{\partial \xi_{3}}
\end{array}\right)+ \\
& +\sum_{m=1}^{3} f_{m}\left(\begin{array}{l}
\frac{1}{h_{1}} \frac{\partial \mathbf{e}_{m}}{\partial \xi_{1}} \bullet \mathbf{e}_{1} \frac{1}{h_{1}} \frac{\partial \mathbf{e}_{m}}{\partial \xi_{1}} \bullet \mathbf{e}_{2} \frac{1}{h_{1}} \frac{\partial \mathbf{e}_{m}}{\partial \xi_{1}} \bullet \mathbf{e}_{3} \\
\frac{1}{h_{2}} \frac{\partial \mathbf{e}_{m}}{\partial \xi_{2}} \bullet \mathbf{e}_{1} \frac{1}{h_{2}} \frac{\partial \mathbf{e}_{m}}{\partial \xi_{2}} \bullet \mathbf{e}_{2} \frac{1}{h_{2}} \frac{\partial \mathbf{e}_{m}}{\partial \xi_{2}} \bullet \mathbf{e}_{3} \\
\frac{1}{h_{3}} \frac{\partial \mathbf{e}_{m}}{\partial \xi_{3}} \bullet \mathbf{e}_{1} \frac{1}{h_{3}} \frac{\partial \mathbf{e}_{m}}{\partial \xi_{3}} \bullet \mathbf{e}_{2} \frac{1}{h_{3}} \frac{\partial \mathbf{e}_{m}}{\partial \xi_{3}} \bullet \mathbf{e}_{3}
\end{array}\right)
\end{aligned}
$$

In this expression two terms exist. The first corresponds to the field derivative and the second corresponds to the derivative of the curvilinear basis. The symbol • indicates dot product. The products $\frac{1}{h_{i}} \frac{\partial \mathbf{e}_{m}}{\partial \xi_{i}} \bullet \mathbf{e}_{j}$ with $j=1,2,3$ project the components of the derivatives on each basis direction.

Regrouping terms and rewriting:

$$
\partial_{i} f_{j}=\frac{1}{h_{i}} \frac{\partial f_{j}}{\partial \xi_{i}}+\sum_{m=1}^{3} f_{m} \frac{1}{h_{i}} \frac{\partial \mathbf{e}_{m}}{\partial \xi_{i}} \bullet \mathbf{e}_{j}
$$

This generic expression is used to compute $\partial_{i} q_{j}$ and $\partial_{i} u_{j}^{a u x}$. The second derivatives $\partial_{i} \partial_{j} f_{k}$ can be obtained as 


$$
\begin{aligned}
\partial_{i} \partial_{j} f_{k}= & \mathbf{e}_{i} \frac{1}{h_{i}} \frac{\partial}{\partial \xi_{i}} \otimes\left[\mathbf{e}_{j} \otimes \mathbf{e}_{k}\left(\frac{1}{h_{j}} \frac{\partial f_{k}}{\partial \xi_{j}}+\sum_{m=1}^{3} f_{m} \frac{1}{h_{j}} \frac{\partial \mathbf{e}_{m}}{\partial \xi_{j}} \bullet \mathbf{e}_{k}\right)\right]= \\
= & \frac{1}{h_{i}} \frac{1}{h_{j}} \frac{\partial^{2} f_{k}}{\partial \xi_{i} \partial \xi_{j}}+\frac{1}{h_{i}} \frac{\partial \frac{1}{h_{j}}}{\partial \xi_{i}} \frac{\partial f_{k}}{\partial \xi_{j}}+\sum_{m=1}^{3} \frac{1}{h_{m}} \frac{\partial f_{k}}{\partial \xi_{m}} \frac{1}{h_{i}} \frac{\partial \mathbf{e}_{m}}{\partial \xi_{i}} \bullet \mathbf{e}_{k}+ \\
& +\sum_{m=1}^{3} \frac{1}{h_{j}} \frac{\partial f_{m}}{\partial \xi_{j}} \frac{1}{h_{i}} \frac{\partial \mathbf{e}_{m}}{\partial \xi_{i}} \bullet \mathbf{e}_{k}+\sum_{m=1}^{3} \frac{1}{h_{i}} \frac{1}{h_{j}} \frac{\partial f_{m}}{\partial \xi_{i}} \frac{\partial \mathbf{e}_{m}}{\partial \xi_{j}} \bullet \mathbf{e}_{k}+ \\
& +\sum_{m=1}^{3} \frac{1}{h_{i}} f_{m} \frac{\partial \frac{1}{h_{j}}}{\partial \xi_{i}} \frac{\partial \mathbf{e}_{m}}{\partial \xi_{j}} \bullet \mathbf{e}_{k}+\sum_{m=1}^{3} \frac{1}{h_{i}} f_{m} \frac{1}{h_{j}} \frac{\partial^{2} \mathbf{e}_{m}}{\partial \xi_{i} \partial \xi_{j}} \bullet \mathbf{e}_{k}+ \\
& +\sum_{l=1}^{3} \sum_{m=1}^{3} \frac{1}{h_{i}} f_{m} \frac{1}{h_{j}}\left(\frac{\partial \mathbf{e}_{m}}{\partial \xi_{l}} \bullet \mathbf{e}_{k}\right)\left(\frac{\partial \mathbf{e}_{l}}{\partial \xi_{i}} \bullet \mathbf{e}_{j}\right)
\end{aligned}
$$

This expression is used for the calculation of $\partial_{i} \partial_{j} u_{k}^{a u x}$. The last derivative term to be obtained is an expression of the form $\partial_{i} f_{j k}$, which is needed in the derivative of the stress and strain fields, $\partial_{i} \sigma_{j k}^{a u x}, \partial_{i} \varepsilon_{j k}^{a u x}$. The result is

$$
\partial_{i} f_{j k}=\frac{1}{h_{i}} \frac{\partial f_{j k}}{\partial \xi_{i}}+\sum_{m=1}^{3} f_{m k} \frac{1}{h_{i}} \frac{\partial \mathbf{e}_{m}}{\partial \xi_{i}} \bullet \mathbf{e}_{k}+\sum_{m=1}^{3} f_{j m} \frac{1}{h_{i}} \frac{\partial \mathbf{e}_{m}}{\partial \xi_{i}} \bullet \mathbf{e}_{j}
$$

In the above expressions, some new terms appear that need to be computed from the level sets. The local coordinates are defined by

$$
\begin{aligned}
\frac{\partial}{\partial \xi_{1}} & =\frac{\partial}{\partial \psi} \\
\frac{\partial}{\partial \xi_{2}} & =\frac{\partial}{\partial \phi}
\end{aligned}
$$

The third component $\frac{\partial}{\partial \xi_{3}}$ does not have a direct correspondence in the level set representation. However it describes the crack direction 3 and measures the crack length $s$ and can be computed with the Jacobian matrix of the transformation, (43). The Jacobian matrix associated with the transformation between the Cartesian system and the curvilinear local system, considering (42) and $\mathbf{e}_{i}$ as the $j$ component of the basis vector $\mathbf{e}_{i}$, is

$$
J=\frac{\partial \xi_{i}}{\partial x_{j}}=\left(\begin{array}{lll}
\left(\mathbf{e}_{1}\right)_{1} & \left(\mathbf{e}_{1}\right)_{2} & \left(\mathbf{e}_{1}\right)_{3} \\
\left(\mathbf{e}_{2}\right)_{1} & \left(\mathbf{e}_{2}\right)_{2} & \left(\mathbf{e}_{2}\right)_{3} \\
\left(\mathbf{e}_{3}\right)_{1} & \left(\mathbf{e}_{3}\right)_{2} & \left(\mathbf{e}_{3}\right)_{3}
\end{array}\right)
$$


The scale factors are computed using the jacobian matrix as

$$
h_{i}^{2}=\sum_{k=1}^{3} J_{k i}^{-1} J_{k i}^{-1}
$$

In contrast to the derivations presented in $[9,10]$, our basis is constructed using averaged finite element gradients evaluated at the nodes. Consequently, the scale factors cannot be assured to be unitary, and therefore all have to be evaluated.

Using the chain derivative rule and the jacobian matrix, the next expression is derived

$$
\begin{gathered}
\frac{\partial \mathbf{e}_{i}}{\partial \xi_{j}}=\sum_{k=1}^{3} \frac{\partial \mathbf{e}_{i}}{\partial x_{k}} \frac{\partial x_{k}}{\partial \xi_{j}}=\sum_{k=1}^{3} \frac{\partial \mathbf{e}_{i}}{\partial x_{k}} J_{k j}^{-1} \\
\frac{\partial \frac{1}{h_{i}}}{\partial \xi_{j}}=\sum_{k=1}^{3} \frac{\partial \frac{1}{h_{i}}}{\partial x_{k}} \frac{\partial x_{k}}{\partial \xi_{j}}=\sum_{k=1}^{3} \frac{\partial \frac{1}{h_{i}}}{\partial x_{k}} J_{k j}^{-1}
\end{gathered}
$$

The information is obtained from the finite element approximation, so the following results are evaluated from nodal values. This fact is explicitly remarked using $\left.\right|_{\mathrm{I}}$

$$
\begin{aligned}
& \frac{\partial \mathbf{e}_{i}}{\partial x_{k}}=\left.\sum_{\mathrm{I}} \frac{\partial N_{\mathrm{I}}}{\partial x_{k}} \mathbf{e}_{i}\right|_{\mathrm{I}} \\
& \frac{\partial \frac{1}{h_{i}}}{\partial x_{k}}=\left.\sum_{\mathrm{I}} \frac{\partial N_{\mathrm{I}}}{\partial x_{k}} \frac{1}{h_{i}}\right|_{\mathrm{I}}
\end{aligned}
$$

In the former equation $\frac{\partial N_{I}}{\partial x_{k}}$ indicates the derivative of the shape function with respect to the respective Cartesian coordinate $x_{k}$. In our work, we use only linear finite element shape functions. In case that higher order finite elements are used, they could be derived from the above expressions.

\section{SIF error indicator and corrector}

The computation of the SIF for non-planar and curved cracks needs a careful treatment [9-11] since the convergence behavior is strongly related to the extraction domain and the crack curvature as shown in [13]. Even for planar cracks in 3-D studies some oscillations may appear in the computation of the SIF associated with the mesh topology [22]. One of the techniques to ensure that the domain integral yields good results is to verify the domain independence by studying the variation with the extraction domain size. However, this 
cannot be used for non-planar and curved cracks as an approximate domain independence is only guaranteed close to the crack front [12]. Furthermore other types of singularities can appear in generic 3-D cracks such as vertex singularities and kink singularities.

The $J$-integral shows a quadratic dependency on the SIF as can be observed in (30). The convergence study realized in [13] shows that it has less dependency on the curvature and better accuracy than the interaction integral. Despite the $J$-integral cannot be used to separate SIF modes, it does not need auxiliary fields, which are one of the error sources when using the interaction integral for curved cracks. The good behavior of the $J$-integral can be used to provide an indication of the quality of the SIF estimation with the interaction integral. The use of this relationship is one of the major contributions of this work. We define, after equation (28), three magnitudes from the SIF obtained using the interaction integral $\left(K_{\mathrm{I}}^{\text {inter }}, K_{\mathrm{II}}^{\text {inter }}\right.$ and $\left.K_{\mathrm{III}}^{\text {inter }}\right)$

$$
\begin{aligned}
& J_{1}^{\text {inter }}=\frac{\left(1-\nu^{2}\right)}{E} K_{\mathrm{I}}^{\text {inter } 2} \\
& J_{2}^{\text {inter }}=\frac{\left(1-\nu^{2}\right)}{E} K_{\mathrm{II}}^{\text {inter } 2} \\
& J_{3}^{\text {inter }}=\frac{1}{2 \mu} K_{\mathrm{III}}^{\text {inter }}{ }^{2}
\end{aligned}
$$

The theoretical relationship between $J$ and the new set of magnitudes defined in equation (47) is clearly

$$
J=J_{1}^{\text {inter }}+J_{2}^{\text {inter }}+J_{3}^{\text {inter }}
$$

but this will only be verified if all the computed SIF match with theoretical value. It gives a straightforward information about the existing differences between the SIFs computed using the interaction integral and the SIFs values associated with the $J$-integral expression. Despite the SIFs associated with the $J$-integral are also an approximated solution, the difference between both sides of Eq. (48) is an indicator of the level of inaccuracy. We define this indicator $r^{\mathrm{SIF}}$ as

$$
r^{\mathrm{SIF}}=\frac{J}{J_{1}^{\text {inter }}+J_{2}^{\text {inter }}+J_{3}^{\text {inter }}}
$$

If the domain integral results were perfectly correct then $r^{\mathrm{SIF}}=1$. Furthermore we propose to use equation (47) to introduce easily a correction for some errors in the computed SIFs, although this approach can only work for situations with little error. The new corrected SIFs will be denoted as $K_{\mathrm{I}}^{\text {corr }}, K_{\mathrm{II}}^{\text {corr }}$ and $K_{\text {III }}^{\text {corr }}$ and are built using the next algorithm: 
If $r^{\mathrm{SIF}} \approx 1$ then we can define the corrected SIFs as

$$
\begin{aligned}
& K_{\mathrm{I}}^{\mathrm{corr}}=r^{\mathrm{SIF}} K_{\mathrm{I}}^{\text {inter }} \\
& K_{\mathrm{III}}^{\mathrm{corr}}=r^{\mathrm{SIF}} K_{\mathrm{II}}^{\text {inter }} \\
& K_{\mathrm{III}}^{\mathrm{corr}}=r^{\mathrm{SIF}} K_{\mathrm{III}}^{\text {inter }}
\end{aligned}
$$

If at a given point, one of the modes is zero, then the following magnitudes are computed from the combinations of the remaining two SIFs

$$
r_{i j}^{\mathrm{SIF}}=\frac{J}{J_{i}^{\text {inter }}+J_{j}^{\text {inter }}}
$$

where $i, j=\mathrm{I}$, II, III and $i \neq j$. If any of the $r_{i j}^{S I F} \approx 1$ then the next correction can be introduced

$$
\begin{aligned}
& K_{i}^{\text {corr }}=r_{i j}^{\mathrm{SIF}} K_{i}^{\text {inter }} \\
& K_{j}^{\text {corr }}=r_{i j}^{\text {SIF }} K_{j}^{\text {inter }} \\
& K_{k}^{\text {corr }}=0
\end{aligned}
$$

where $i, j, k=\mathrm{I}$, II, III and $i \neq j, j \neq k$ and $i \neq k$.

Another possibility is that, at a given point, only one mode exists. Then we can define $r_{k}^{\mathrm{SIF}}$ which involves only one mode:

$$
r_{k}^{\mathrm{SIF}}=\frac{J}{J_{k}^{\text {inter }}}
$$

If any $r_{k}^{\mathrm{SIF}}$ verifies $r_{k}^{\mathrm{SIF}} \approx 1$ then the correction is defined by

$$
\begin{aligned}
& K_{k}^{\text {corr }}=r_{k}^{\mathrm{SIF}} K_{k}^{\text {inter }} \\
& K_{l}^{\text {corr }}=0
\end{aligned}
$$

As before $k, l=\mathrm{I}$, II, III and $k \neq l$. If none of the former conditions verifies then a correction is not possible and the results of the interaction integral should be discarded. This result may indicate either that the mesh is not refined enough for achieving a correct description of the crack or the extraction domain is too big for the crack curvature.

In order to use $r^{S I F}$ as a correction factor, we propose in this work a limiting value in the range of $[0.95,1.05]$ of $r^{S I F}$. The correction proposed has to be considered carefully and only used in regions where some errors due to the mesh or curvature can be expected. 


\section{Reference problems and numerical result}

The main complication about the selection of reference problems in three dimensions is the lack of problems with known analytical solutions. Problems where the solution is available correspond to infinite domains. Three problems have been selected for the numerical verification: the extruded arc crack, the elliptical crack under traction and the elliptical crack under shear. The arc crack was also studied in [10] and the exact SIFs for elliptic crack problems can be found in [21]. The effect of the curvature in a similar elliptic crack has also been analyzed in $[9,11]$. The finite element mesh is formed using regular hexahedron linear elements in the domain where the integration is performed.

We use our proposal for the interaction integral formulation, as it has been shown in [13] that it improves the estimation. Our proposal for the orthogonal basis is also used. The first objective is to consider the effect of the curvilinear correction in the gradients of the auxiliary fields and $q$ in XFEM, since it improves the solution in standard FE as reported in $[9,10]$. Finally our proposed $J$-based error indicator will be used to evaluate the quality of the results.

\subsection{Arc crack analysis}

The problem considered is an arc crack in an infinite plate subjected to equibiaxial tension. The geometry of the crack is defined by the radius $R_{c}$ and the angle $\beta$, with values $R_{c}=1$ and $\beta=\pi / 2$, as shown in Figure 8 . We will consider a finite extrusion of this two dimension model, applying symmetry boundary conditions to simulate an infinite thickness plate. The domain used for the XFEM analyses is a finite portion defined by a width $w=2$, a height $h=4$ and a thickness $t=1$. The analytical stress field solution and symmetry conditions are imposed on the boundary of the finite domain in order to make the model equivalent to the infinite domain problem. The analytical solution to this problem is given in [25]. The reference solution for the SIFs is:

$$
\begin{aligned}
& K_{\mathrm{I}}^{e x}=\sigma_{\infty}(\pi a)^{\frac{1}{2}} \frac{\cos \left(\frac{\beta}{2}\right)}{1+\sin ^{2}\left(\frac{\beta}{2}\right)} \\
& K_{\mathrm{II}}^{e x}=\sigma_{\infty}(\pi a)^{\frac{1}{2}} \frac{\sin \left(\frac{\beta}{2}\right)}{1+\sin ^{2}\left(\frac{\beta}{2}\right)} \\
& K_{\mathrm{III}}^{e x}=0
\end{aligned}
$$

where $a=R_{c} \sin (\beta)$ is related to the crack length and $\sigma_{\infty}$ is the applied remote load. The following convergence rate study for this reference problem is carried out using a mesh sequence with regular hexahedron elements with 
uniform refinement. The mesh sequence used for the analysis is built using elements with a side length defined by the following series:

$h=\left(\frac{1}{8}, \frac{1}{16}, \frac{1}{20}, \frac{1}{32}, \frac{1}{40}\right) R_{c}$.

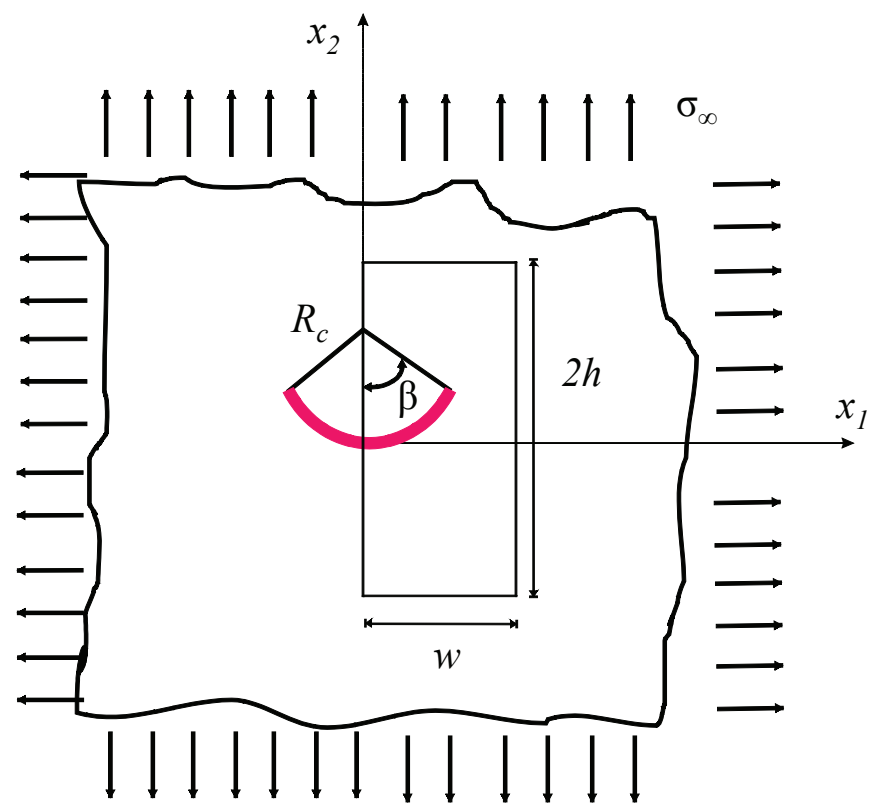

Fig. 8. Section for the problem of the extruded arc crack in an infinitely extended plate subjected to equibiaxial tension.

The SIF distribution along the crack front for $K_{\mathrm{I}}, K_{\mathrm{II}}$ and $J$ with an extraction domain defined by $R_{q}=0.1 R_{c}$ and length $S_{q}$ equal to the element size, is shown in Figures 9, 10 and 11. Gosz [10], achieves a relative minimum error of $0.4 \%$ with quadratic elements and an element size $h=\frac{1}{500} R_{c}$. The minimum relative error in our approximation is less than the $0.7 \%$ for an element size of $h=\frac{1}{40} R_{c}$. This result shows the accuracy of our proposal.

In Figs. 9, 10, 11, a slightly different result is found for elements at the end of the crack front when compared to the solution at the inner part of the crack front. This is due to the subdivision of the hexaedra for integration purposes, which results in a different subdomain topology at the elements that interesect the side boundaries of the extruded domain.

The error convergence rates vs. the element size $h$ for $K_{\text {I }}, K_{\text {II }}$ and $J$ are shown in Figures 12, 13 and 14 respectively. The optimum convergence rate with linear elements is 1.0. The convergence results are close to the expected value but show some differences with respect to the optimum value. The main reason of this divergence is the topological variation of the extraction domain. This effect was described above, see Fig. 6.

We now study the curvilinear correction using the results for the element size $h=\frac{1}{40} R_{c}$ (i.e. the most accurate results shown in Figures 9, 10 and 11). 


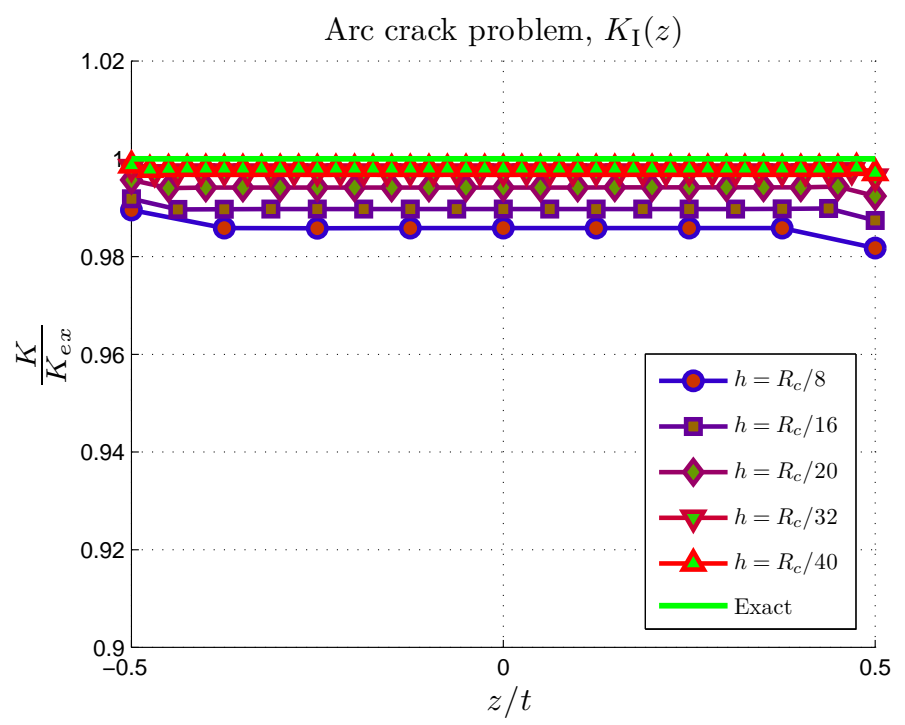

Fig. 9. $K_{\mathrm{I}}$ distribution along the crack front for the three-dimensional arc crack reference problem.

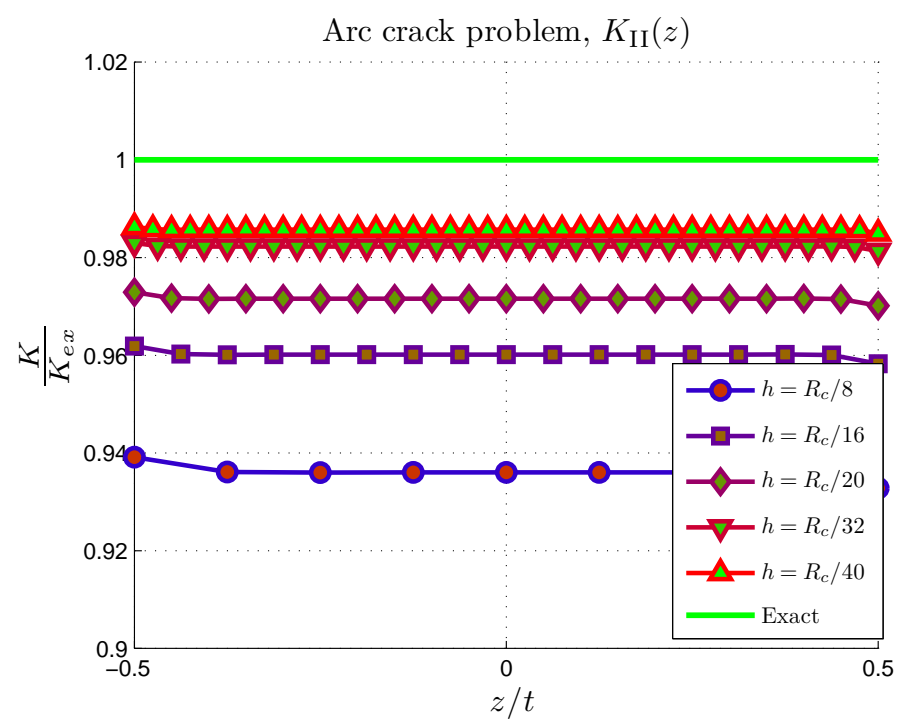

Fig. 10. $K_{\text {II }}$ distribution along the crack front for the three-dimensional arc crack reference problem.

The effect of taking into account the curvilinear gradients following equations (39), (40) and (41) is shown in Figures 15, 16 and 17 for $K_{\mathrm{I}}, K_{\mathrm{II}}$ and $J$ respectively. Although the result for $J$-integral seems more accurate without the curvilinear correction, it is a very small difference and does not modify the error convergence rate. Fig. 18 shows the behavior of $K_{\mathrm{I}}$ for the whole mesh sequence without considering the curvilinear correction (that can be compared with the results presented in Fig. 9). It can be observed that it does not converge to the theoretical value. From these results we can conclude that the inclusion of the curvilinear gradients as in Section 5 is necessary to 


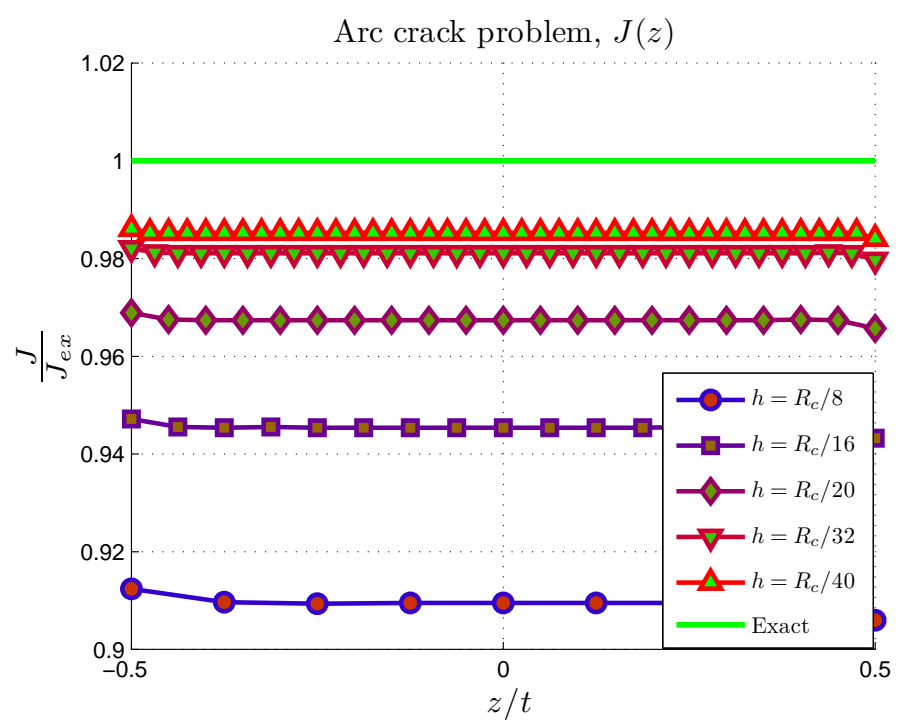

Fig. 11. $J$ distribution along the crack front for the three-dimensional arc crack reference problem.

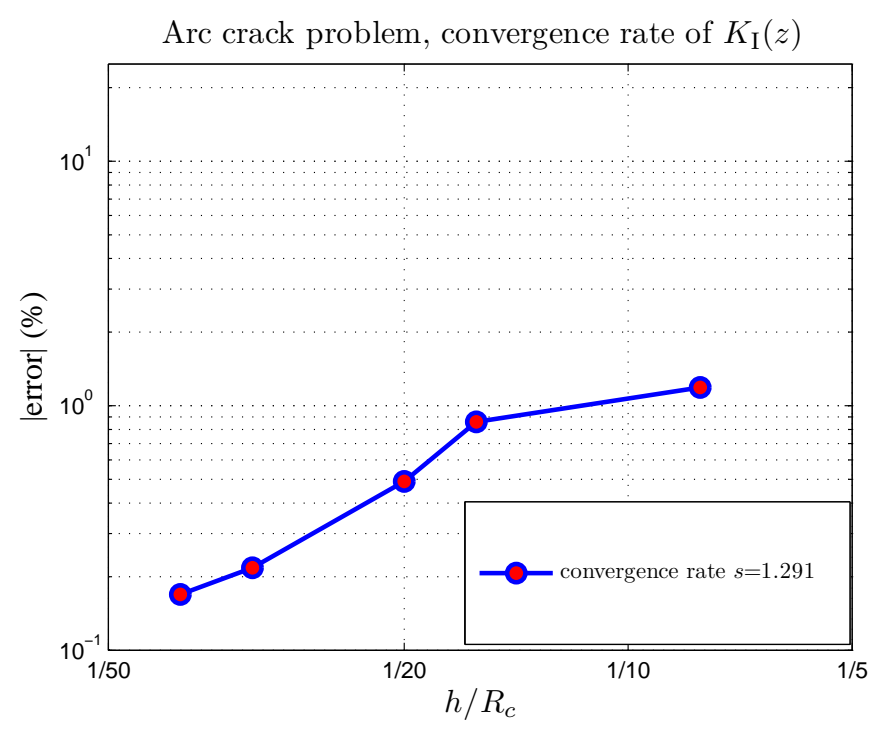

Fig. 12. Convergence rate of the average error in $K_{\mathrm{I}}$ for the three dimensional extruded arc crack problem.

achieve a correct behavior with the interaction integral when studying non planar cracks.

Although the results seem to be reasonably good, we will verify the behavior using the $J$ based SIF error indicator. The results for $r^{\text {SIF }}$ according to Section 6 are presented in Figure 19, where the expected loss of accuracy near the boundary and the element size convergence effect can be observed. Note that the poor results for $r^{\mathrm{SIF}}$ in Fig. 19 near $|z / t|=0.5$ are due to the existence of a spurious mode III that theoretically should not exist, but that appears when computing the numerical solution. Applying the correction to the re- 


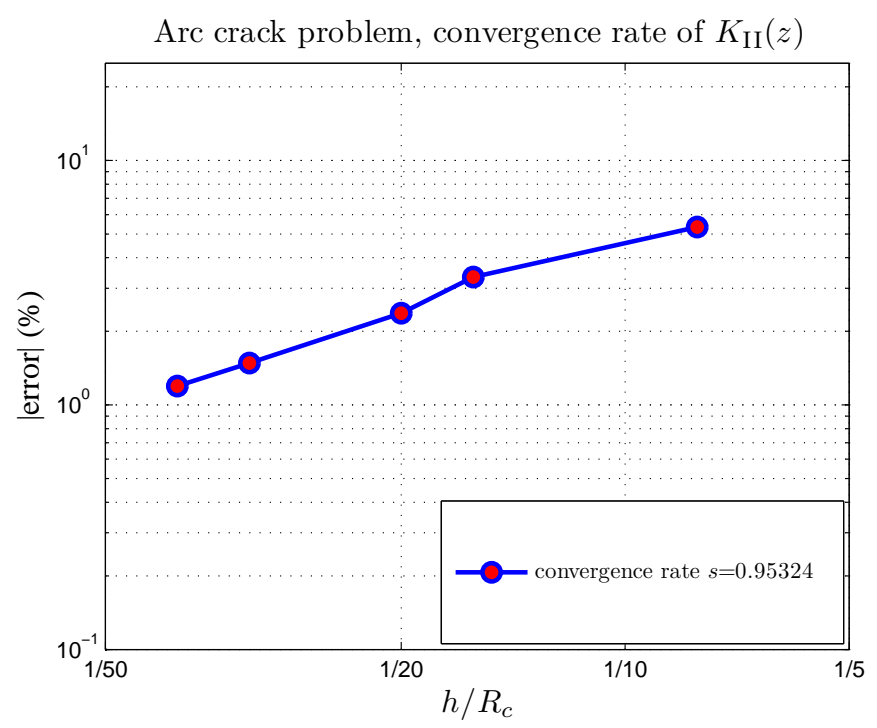

Fig. 13. Convergence rate of the average error in $K_{\text {II }}$ for the three dimensional extruded arc crack problem.

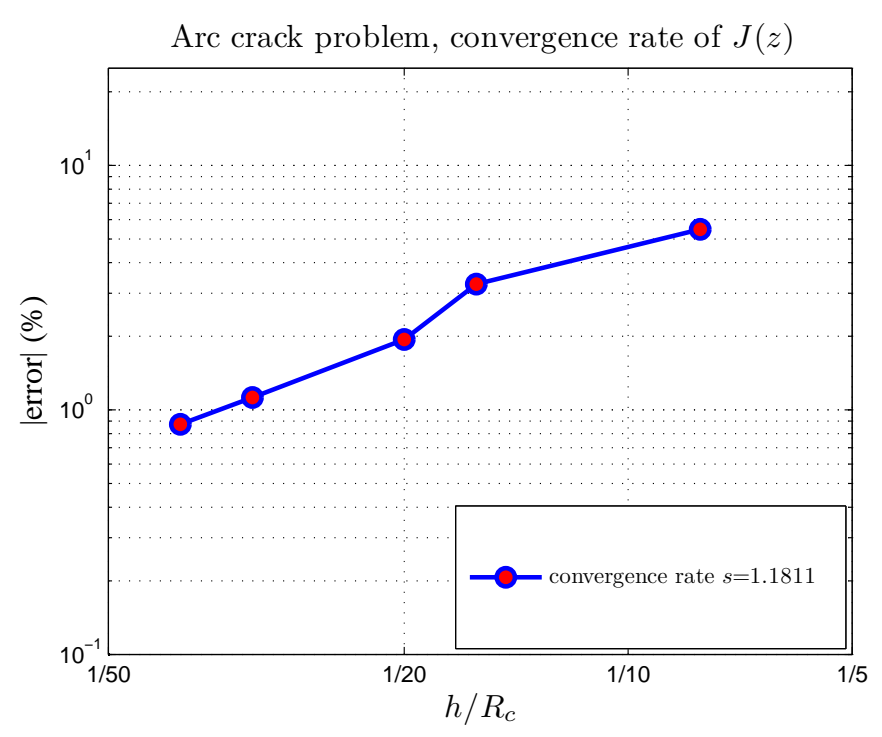

Fig. 14. Convergence rate of the average error in $J$ for the three dimensional extruded arc crack problem

sults of the finest mesh and comparing with the original values we obtain the improved SIF shown in Figures 20 and 21.

\subsection{Elliptic crack analysis}

The elliptic crack model considers an infinite solid under remote constant stress. Two stress states are analyzed: a uniform remote tensile traction, $\sigma_{\infty}$, which produces mode I, and a uniform remote shear, $\tau_{\infty}$, which produces mode 


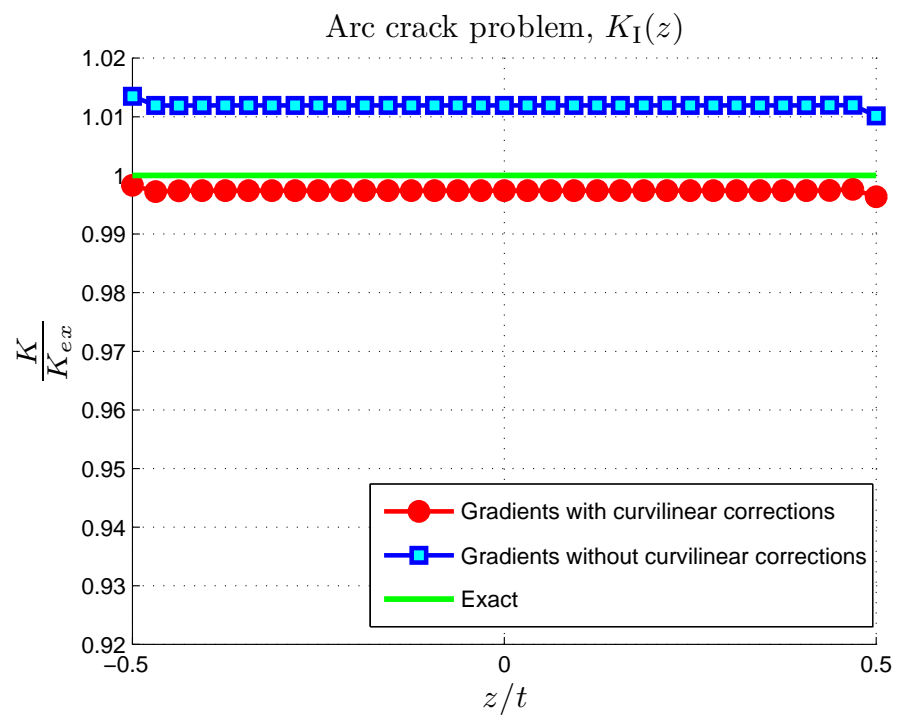

Fig. 15. Comparison of the effect of the curvilinear gradients consideration in the interaction integral. $K_{\mathrm{I}}$ for the three dimensional extruded arc crack problem.

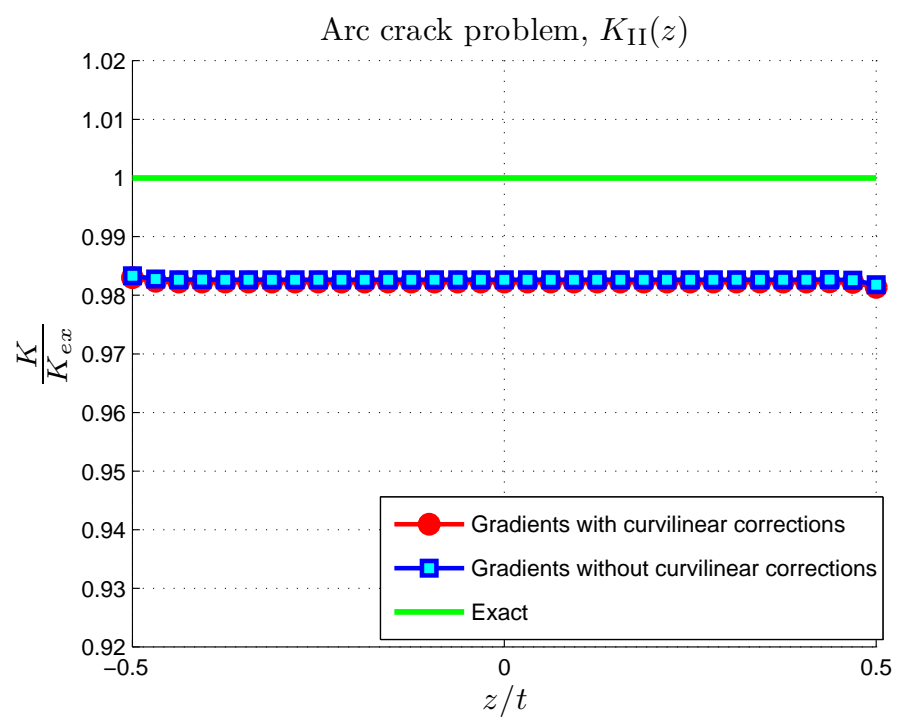

Fig. 16. Comparison of the effect of the curvilinear gradients consideration in the interaction integral. $K_{\mathrm{II}}$ for the three dimensional extruded arc crack problem.

II and III. The crack geometry and applied loads can be observed in Figure 22.

The analytical SIF values for the selected elliptic crack configuration can be found in several references, such as $[9,21,26-28]$ : 


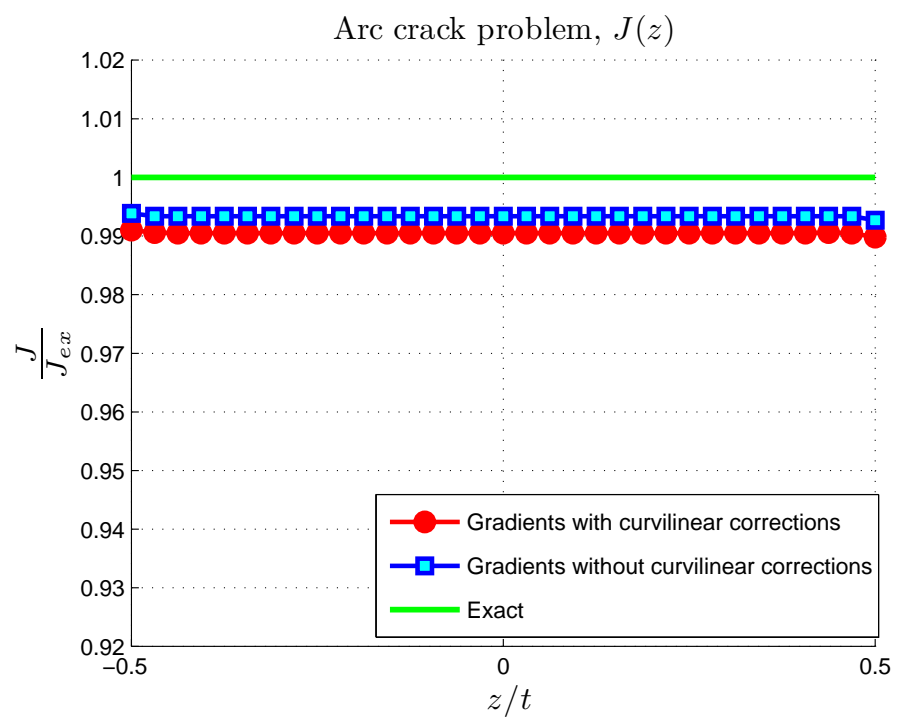

Fig. 17. Comparison of the effect of the curvilinear gradients consideration in the $J$-integral. $J$ for the three dimensional extruded arc crack problem.

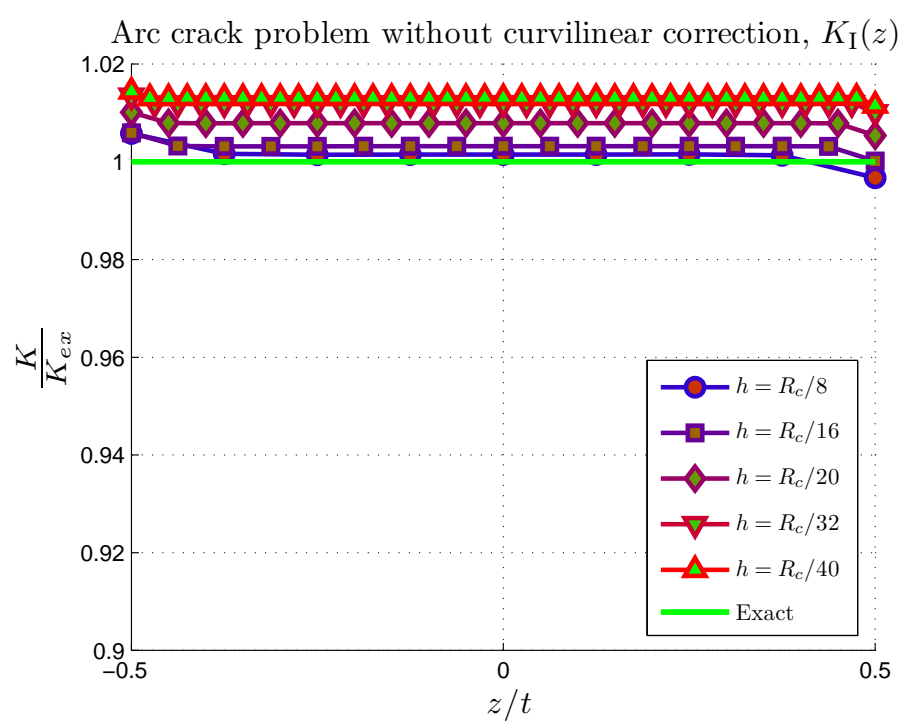

Fig. 18. $K_{\mathrm{I}}$ distribution along the crack front for the three-dimensional arc crack reference problem. The curvilinear gradient correction is not applied.

$$
\begin{aligned}
K_{\mathrm{I}}^{e x} & =\frac{\sigma_{\infty}}{E^{\prime}\{k\}}\left(\frac{\pi c}{a}\right)^{\frac{1}{2}} f(a, c, \varphi) \\
K_{\mathrm{II}}^{e x} & =\left(\frac{\pi a^{3}}{c}\right)^{\frac{1}{2}} \frac{k^{2} \tau_{\infty} \sin \varphi}{\left[\left(k^{2}+\nu\left(k^{\prime}\right)^{2}\right) E\{k\}+\left(k^{\prime}\right)^{2} K\{k\}\right] f(a, c, \varphi)} \\
K_{\mathrm{III}}^{e x} & =4(\pi a c)^{\frac{1}{2}} \frac{-(1-\nu) k^{2} \tau_{\infty} \cos \varphi}{\left[\left(k^{2}-\nu\right) E\{k\}+\nu\left(k^{\prime}\right)^{2} K\{k\}\right] f(a, c, \varphi)}
\end{aligned}
$$




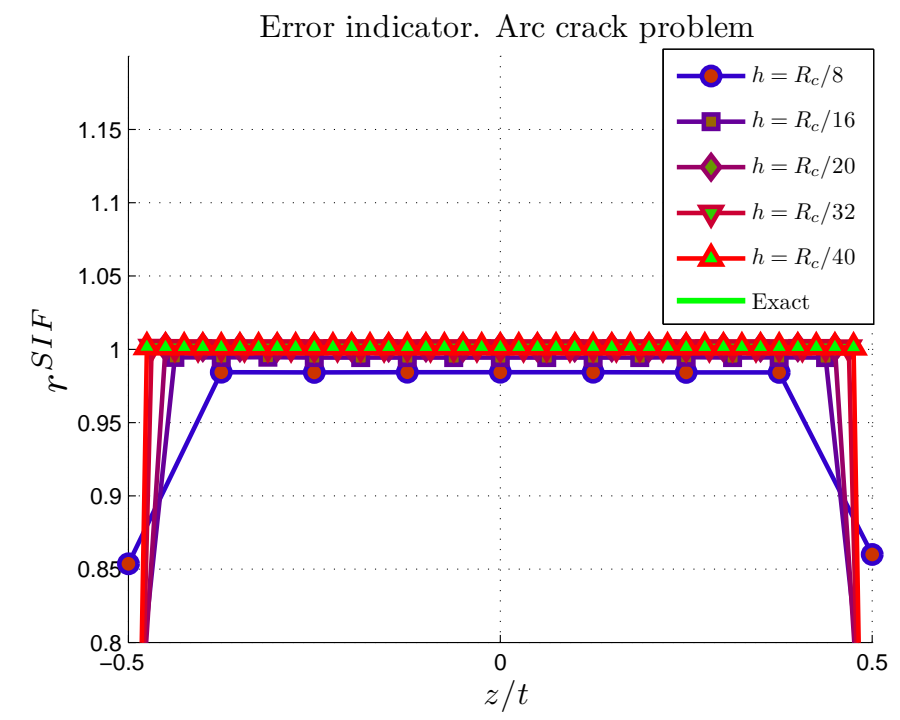

Fig. 19. Error indicator distribution for the mesh sequence along the crack front for the three-dimensional arc crack problem.

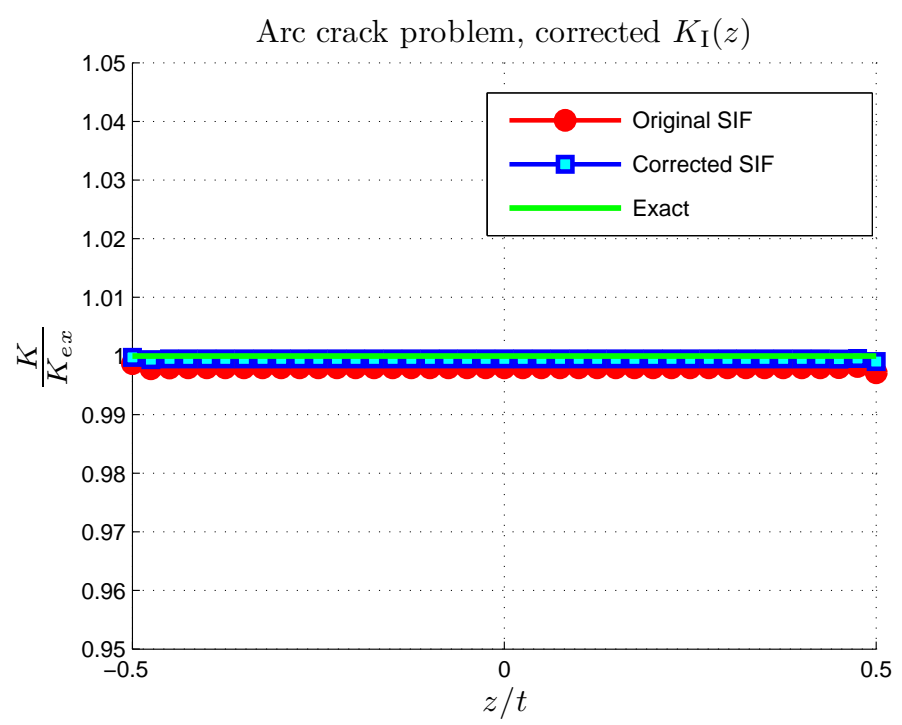

Fig. 20. $K_{\mathrm{I}}$ along the crack front for the three-dimensional arc crack problem. Effect of SIF correction using the relationship between $J$-integral and interaction integral.

where: 


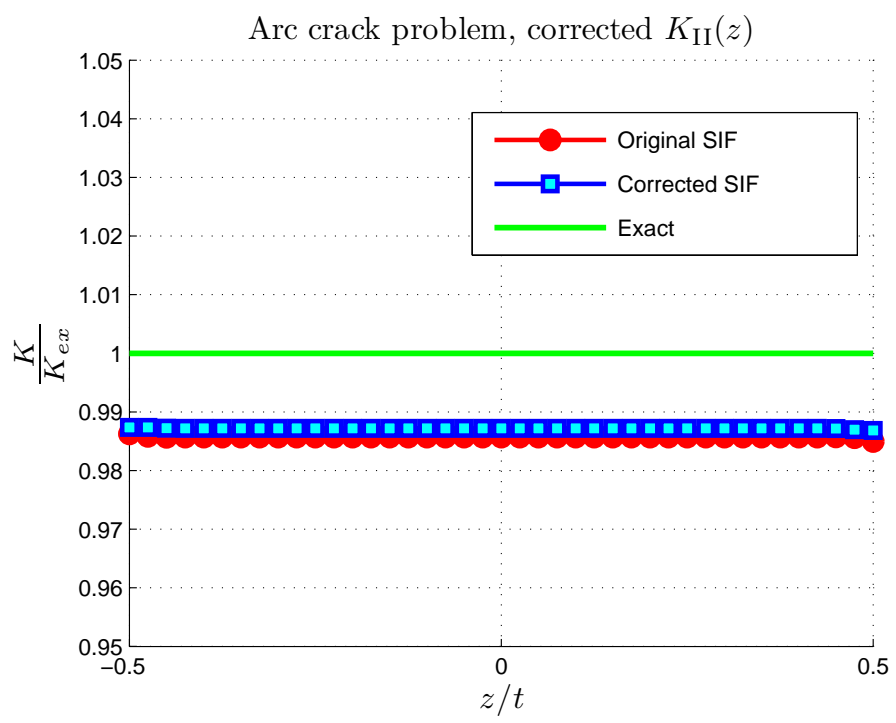

Fig. 21. $K_{\text {II }}$ along the crack front for the three-dimensional arc crack problem. Effect of SIF correction using the relationship between $J$-integral and interaction integral.

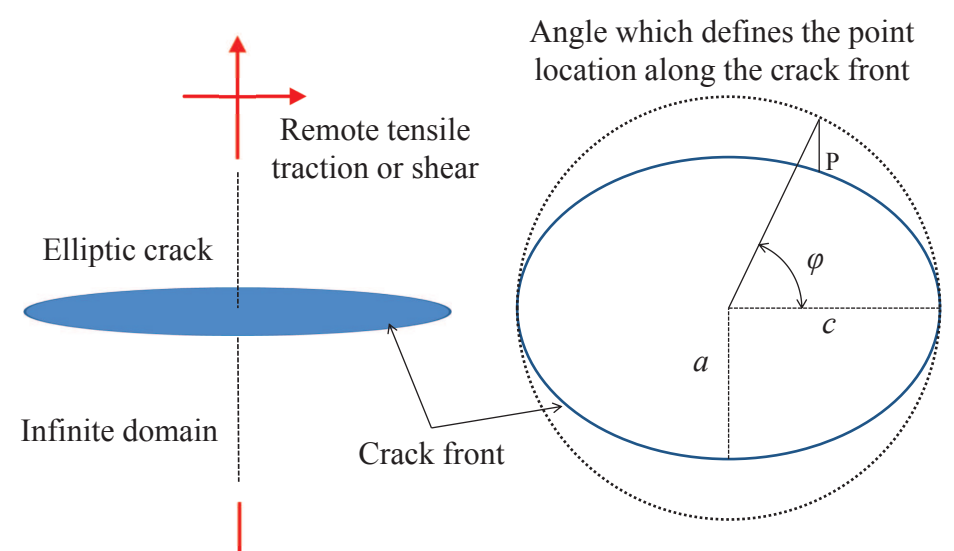

Ellipse geometry

Fig. 22. Elliptic crack in infinite solid. Elliptic angle $\varphi$ definition.

$$
\begin{aligned}
f(a, c, \varphi) & =\left(a^{2} \sin ^{2} \varphi+c^{2} \cos ^{2} \varphi\right)^{\frac{1}{4}} \\
k & =\left(1-\frac{a^{2}}{c^{2}}\right)^{\frac{1}{2}} \\
k^{\prime} & =\frac{a}{c} \\
E\{k\} & =\int_{0}^{\frac{\pi}{2}}\left(1-k^{2} \sin ^{2} \varphi\right)^{\frac{1}{2}} \mathrm{~d} \varphi \\
E^{\prime}\{k\} & =\frac{c}{a} E\{k\} \\
K\{k\} & =\int_{0}^{\frac{\pi}{2}} \frac{\mathrm{d} \varphi}{\left(1-k^{2} \sin ^{2} \varphi\right)^{\frac{1}{2}}}
\end{aligned}
$$


The numerical model is a plate whose dimensions are $t=10 a, w=10 \mathrm{c}$ and $h=10 a$. Symmetry conditions have been applied to define only a quarter of the domain. The ratio between the plate dimensions and crack dimensions is deemed sufficient to model an infinite behavior. The material properties are $E=10^{4}$ and $\nu=0.3$.

An hexahedron element mesh is used, with a regular topology in a parallepipedic region centered at the ellipse center with dimensions $\frac{5 c}{6} \times \frac{2 c}{3} \times \frac{4 c}{3}$, as observed in Figure 23. The mesh sequence used in the analysis is defined by the element size $h=\left(\frac{1}{6}, \frac{1}{12}, \frac{1}{24}, \frac{1}{36}\right) a$. The element size used is fine enough to detect the potential influence of the curvature on the results.

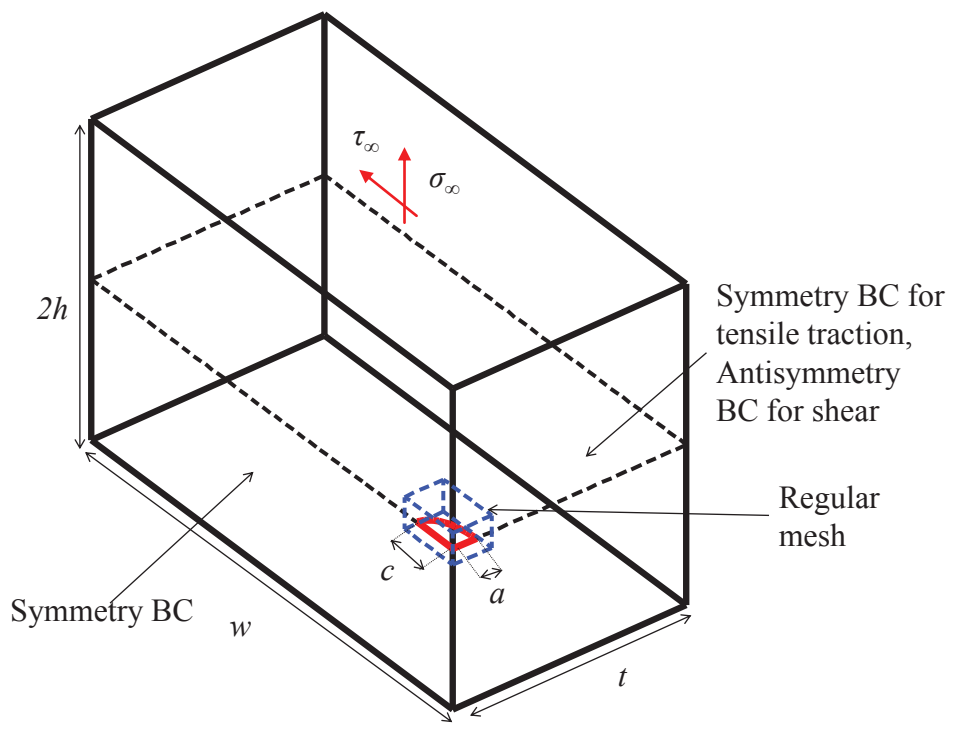

Fig. 23. Elliptic crack geometric model

In this problem, the extraction domain is defined by the dimensions $S_{q}=$ $0,033 c$ and $R_{q}=0,1 c$. This selection is motivated by the crack dimensions and relative topology between the crack and the mesh. The elliptic crack results for the pure traction state are shown in Figures 24 and 25 for $K_{\mathrm{I}}$ and $J$. Results for a pure shear loading are shown in Figures 26, 27 and 28 for $K_{\text {II }}$, $K_{\text {III }}$ and $J$, respectively. It is possible to observe that the results are improved with the mesh refinement, even under the effects of the topological oscillations of the extraction domain shown in Figures 6 and 7. However a loss of accuracy appears near the boundary.

This effect is due to problems in the extraction domain and local basis definition related with the topological relationship between the crack geometry and the mesh topology. Oscillations are mainly produced by the fact that the extraction domain is not convex.

The error order observed in the SIF for the finest mesh is of the same order of the errors presented in the bibliography for finite element studies with a 
mesh adapted to the crack and smaller element size. For the model under pure tensile traction an error of $2 \%$ is reported in [9] for an element size of $h=\frac{a}{200}$ and an error or about 1\% is reported [11] for an element size of $h=\frac{a}{60}$. Our error order is about $2 \%$ with an element size of $h=\frac{a}{36}$. Further differences exist in the model for remote shear. In [11] is reported an error of about $2 \%$ for an element size of $h=\frac{a}{60}$ and our error order, without considering the region affected by the boundaries inaccuracies, is about $5 \%$ for an element size of $h=\stackrel{a}{36}$.

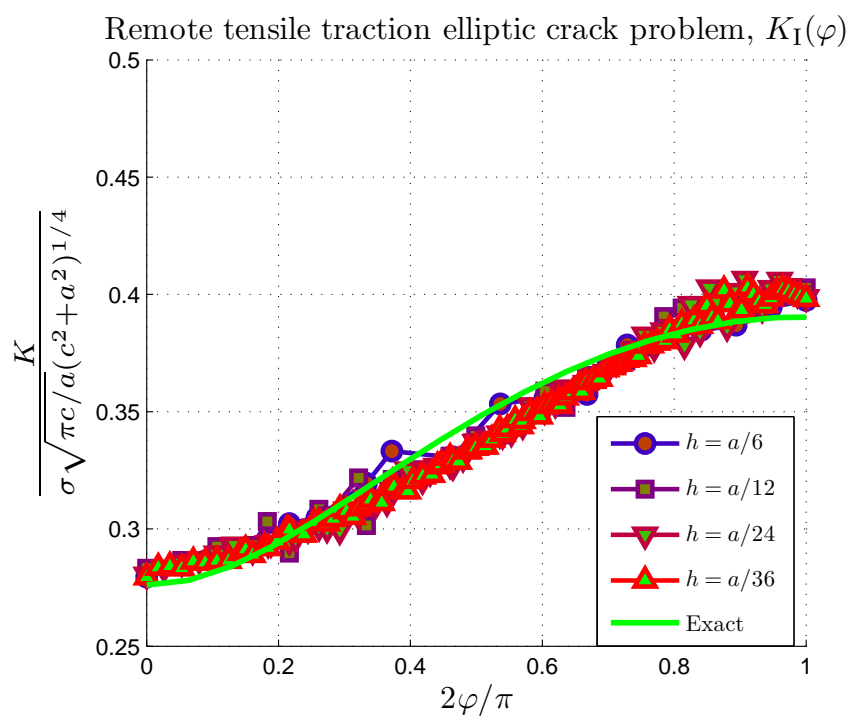

Fig. 24. $K_{\mathrm{I}}$ distribution along the crack front for different element sizes. Elliptic crack under remote tensile traction.

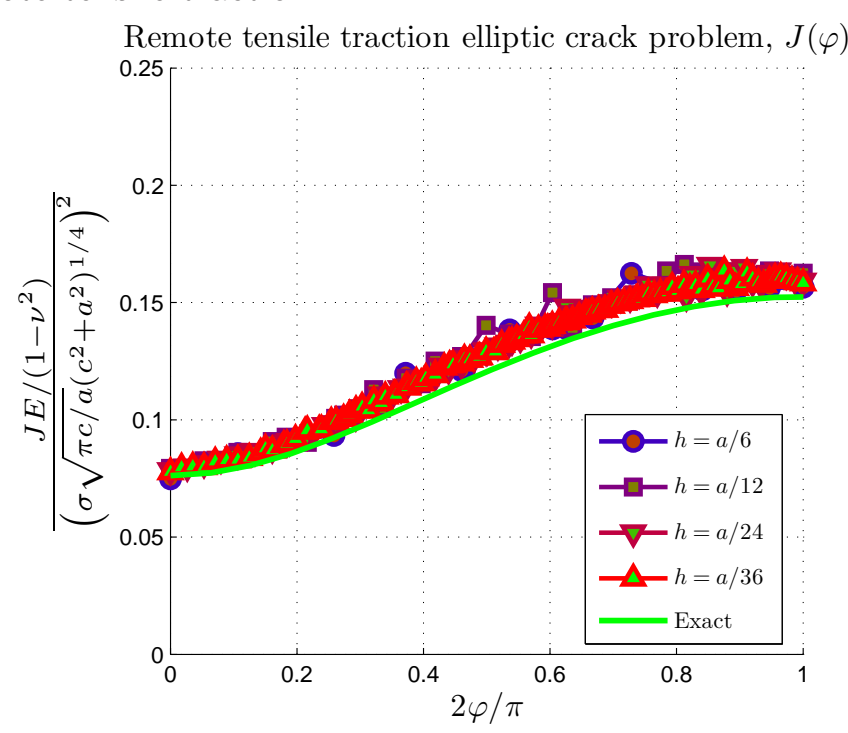

Fig. 25. $J$ distribution along the crack front for different element sizes. Elliptic crack under remote tensile traction.

The effect of curvilinear correction of the gradients in the domain integrals is shown in Figures 29 and 30 for $K_{\mathrm{I}}$ and $J$ for the crack under remote tensile 


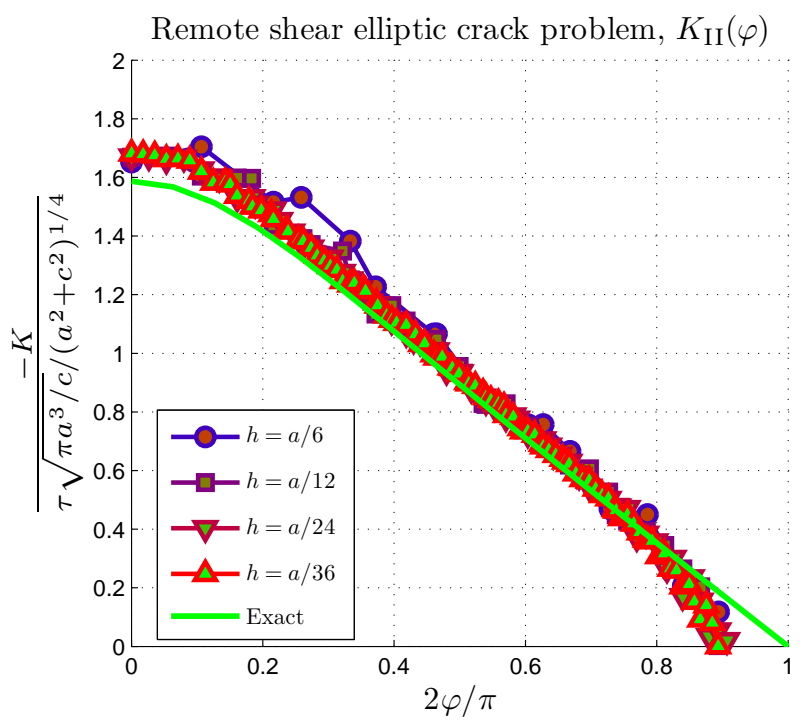

Fig. 26. $K_{\text {II }}$ distribution along the crack front for different element sizes. Elliptic crack under remote shear.

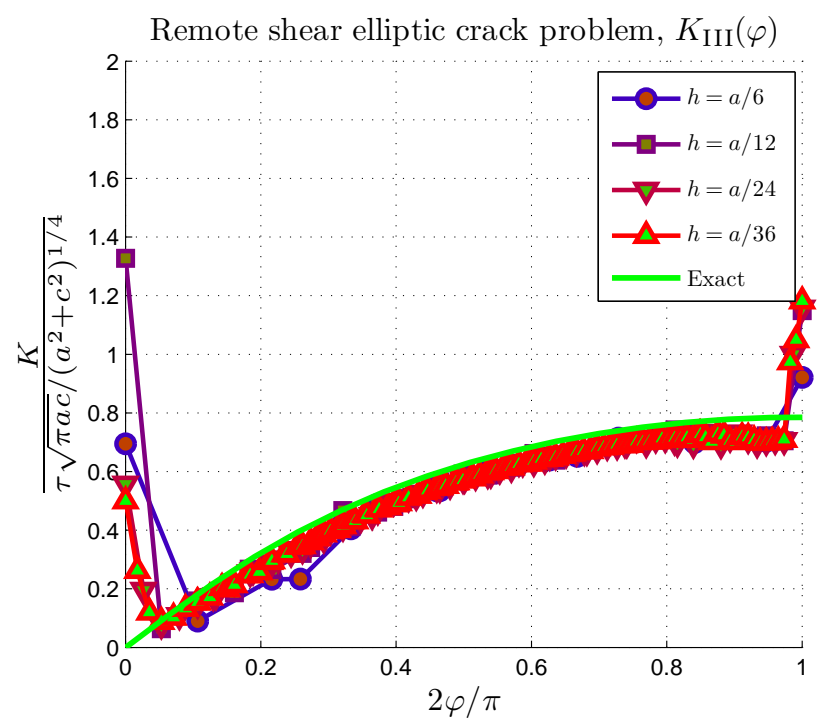

Fig. 27. $K_{\text {III }}$ distribution along the crack front for different element sizes. Elliptic crack under remote shear.

traction for the finest element size, $h=\frac{a}{36}$. It is also shown in Figures 31, 32 and 33 for $K_{\text {II }}, K_{\text {III }}$ and $J$ for the crack under remote shear. It can be observed that the introduction of the curvilinear correction improves the results close to $\varphi=0$. As expected the improvement appears in the region with greater curvature. The same behavior is presented in [9] for the analytical introduction of the curvature terms using finite elements.

The defined extraction domain and the finest element size used can yield good results but do not allow to achieve the accuracy and optimum behavior, because it is still too coarse to describe some of the curvature effects for 


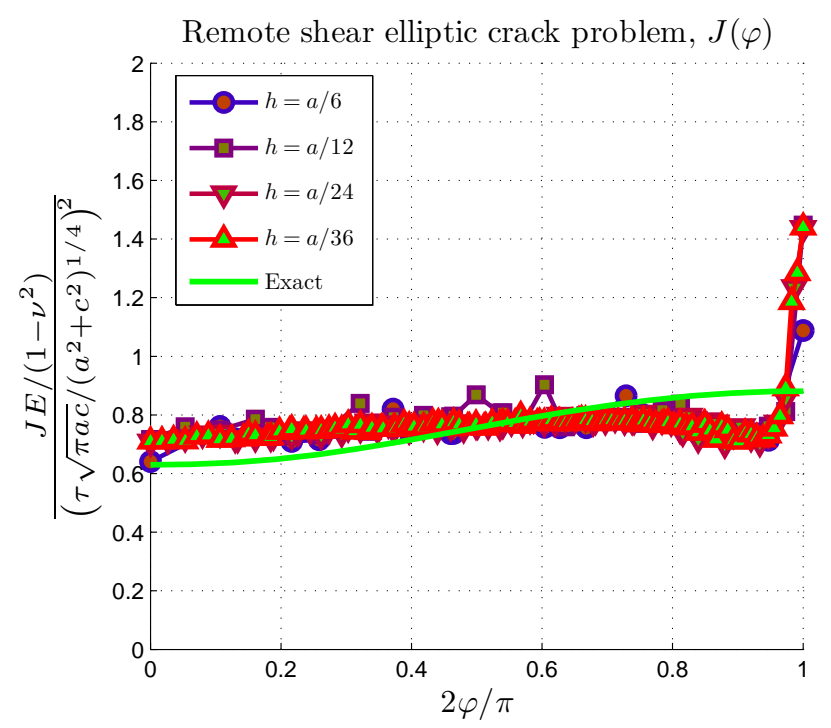

Fig. 28. $J$ distribution along the crack front for different element sizes. Elliptic crack under remote shear.

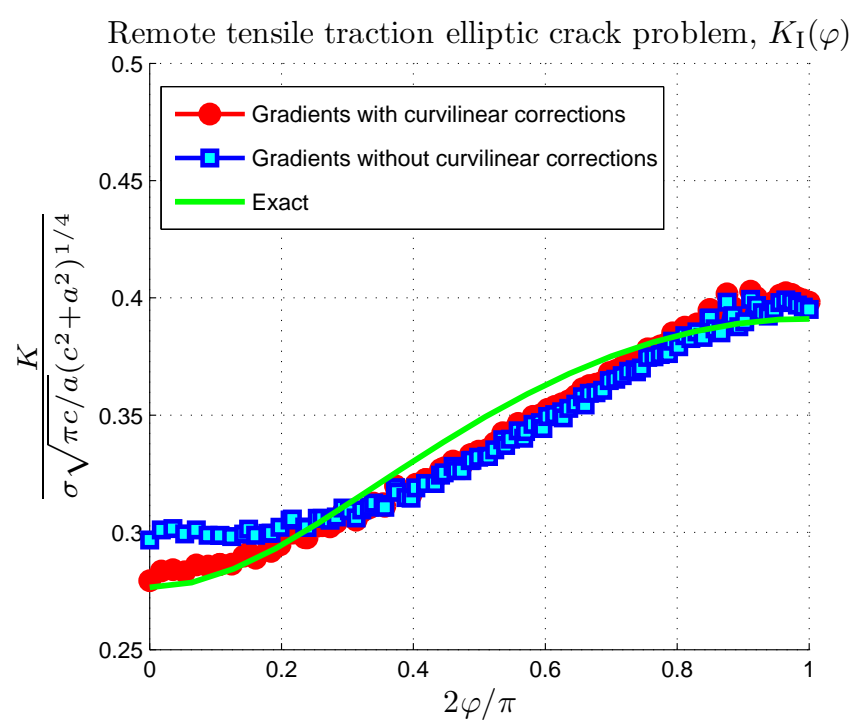

Fig. 29. Comparison of the effect of the curvilinear gradients consideration in the interaction integral. $K_{\mathrm{I}}$ for elliptic crack under remote tensile traction.

small $\varphi$, as shown in [13] for 2-D. In addition, the convexity and lack of smothness of the extraction domain produce oscillations that affect the quality of the solution. In order to verify the behaviour of the proposed methodology with non-structured meshes, we have generated new meshes with a random modification of the nodal coordinates in the mesh corresponding to an element size $h=\frac{a}{12}$. The effect of curvilinear correction of the gradients in the domain integrals for the elliptic crack in a non-structured mesh is shown in Fig. 34 for $K_{\text {I }}$ (remote tensile traction) and in Figs. 35 and 36 for $K_{\text {II }}$ and $K_{\text {III }}$ for the crack under remote shear. Although some oscillations appear in the solution, the improvement can be observed in the region with greater curvature. 


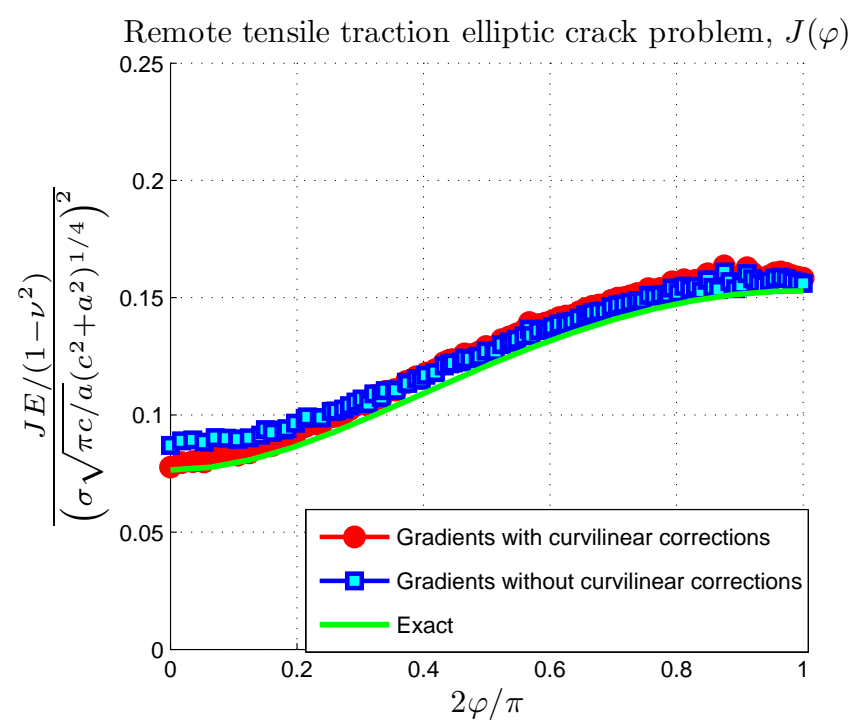

Fig. 30. Comparison of the effect of the curvilinear gradients consideration in the domain integral. $J$ for elliptic crack under remote tensile traction.

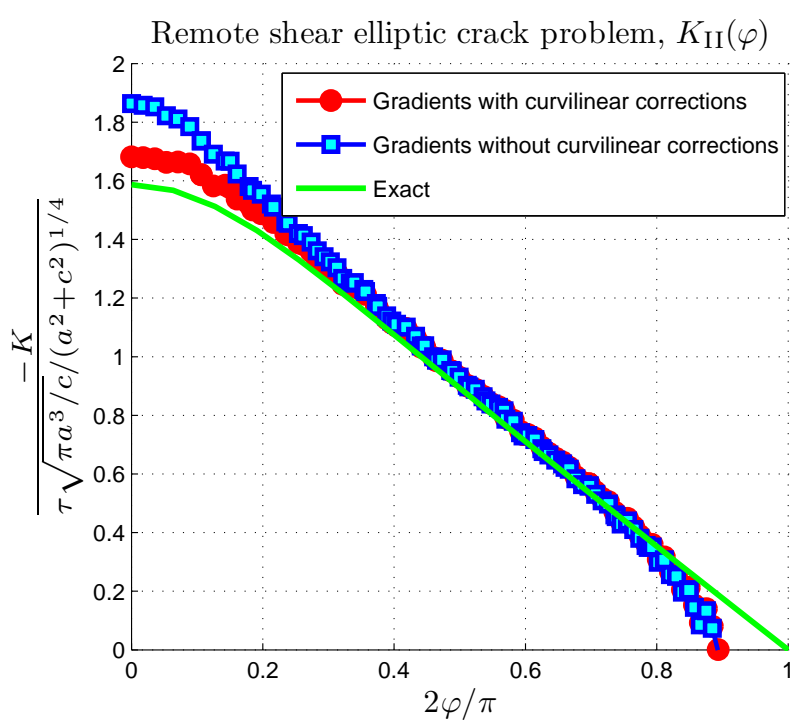

Fig. 31. Comparison of the effect of the curvilinear gradients consideration in the interaction integral. $K_{\text {II }}$ for elliptic crack under remote shear.

The lack of accuracy is checked using the proposed error indicator. The result for the elliptic crack under remote tensile traction is shown in Figure 37 and for remote shear in Figure 38. The error indicator shows that a mismatch of about $15 \%$ exists. The mismatch of $15 \%$ provided by the error indicator should not be directly related with the true error level (which in this case is about $5 \%$ ). The error indicator simply detects the regions where greater differences exist between the crack and the mesh relative topologies, and where higher error is expected. This error indicator suggests that a mesh refinement or an adaptation of the mesh to the crack topology will improve the solution 


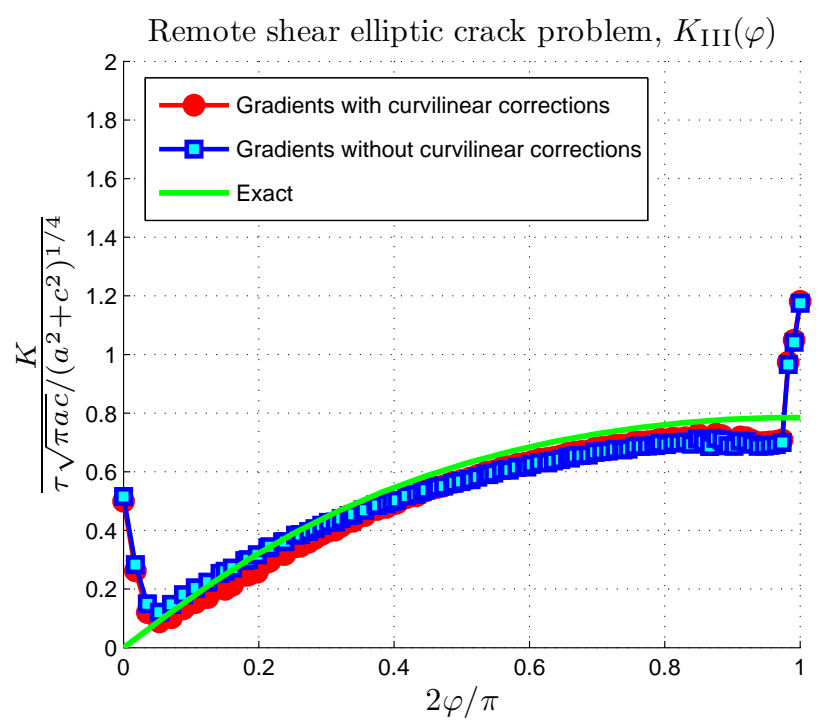

Fig. 32. Comparison of the effect of the curvilinear gradients consideration in the interaction integral. $K_{\text {III }}$ for elliptic crack under remote shear.

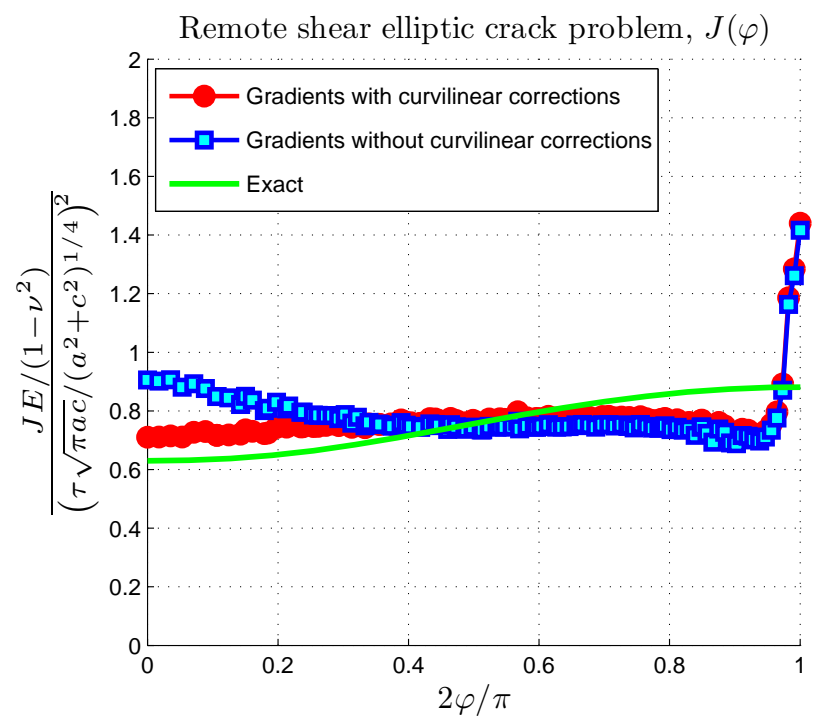

Fig. 33. Comparison of the effect of the curvilinear gradients consideration in the domain integral. $J$ for elliptic crack under remote shear. 


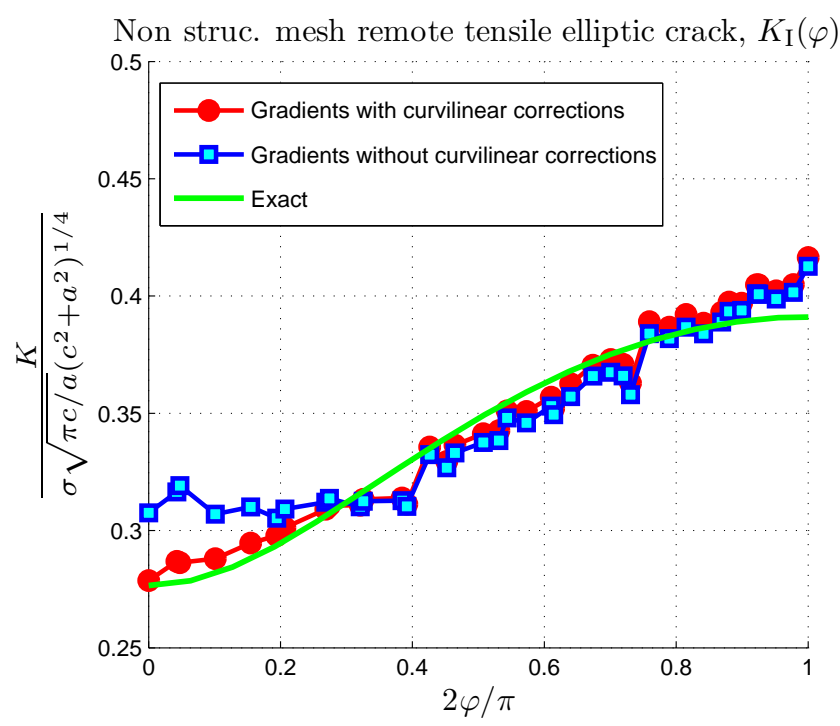

Fig. 34. Comparison of the effect of the curvilinear gradients consideration in the interaction integral. $K_{\mathrm{I}}$ for elliptic crack under remote tensile traction using a non structured mesh.

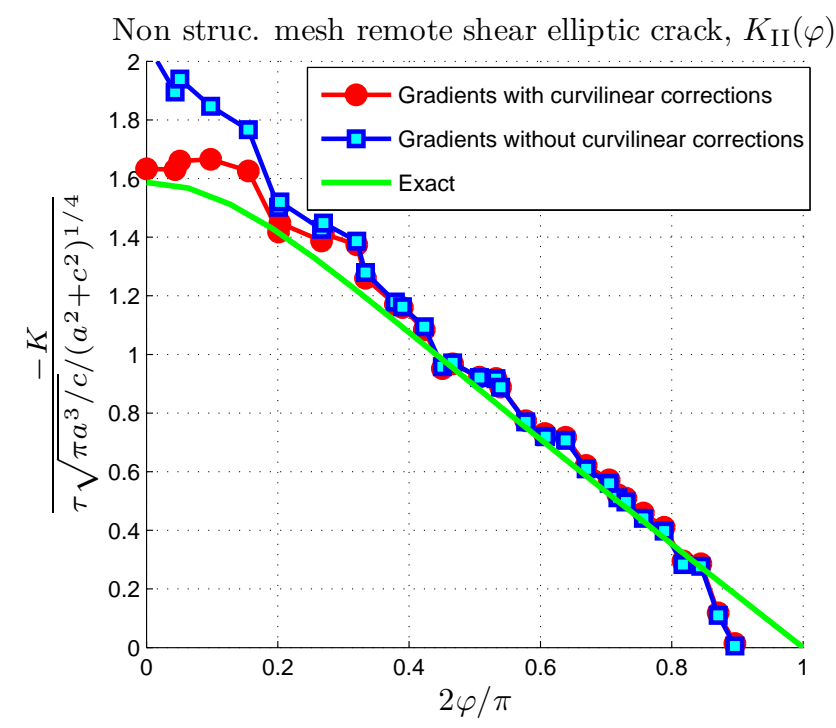

Fig. 35. Comparison of the effect of the curvilinear gradients consideration in the interaction integral. $K_{\text {II }}$ for elliptic crack under remote shear using a non structured mesh. 


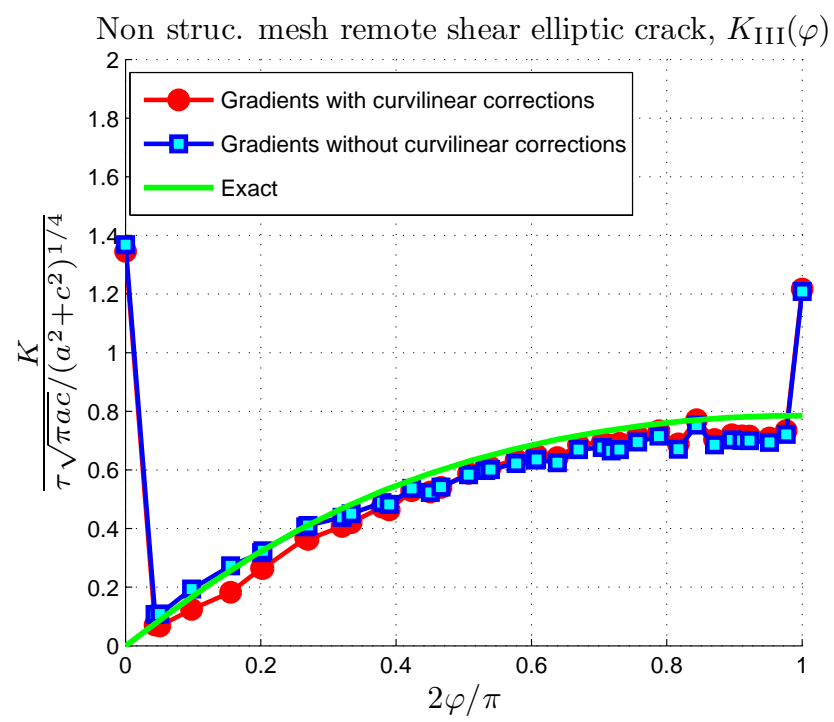

Fig. 36. Comparison of the effect of the curvilinear gradients consideration in the interaction integral. $K_{\text {III }}$ for elliptic crack under remote shear using a non structured mesh.

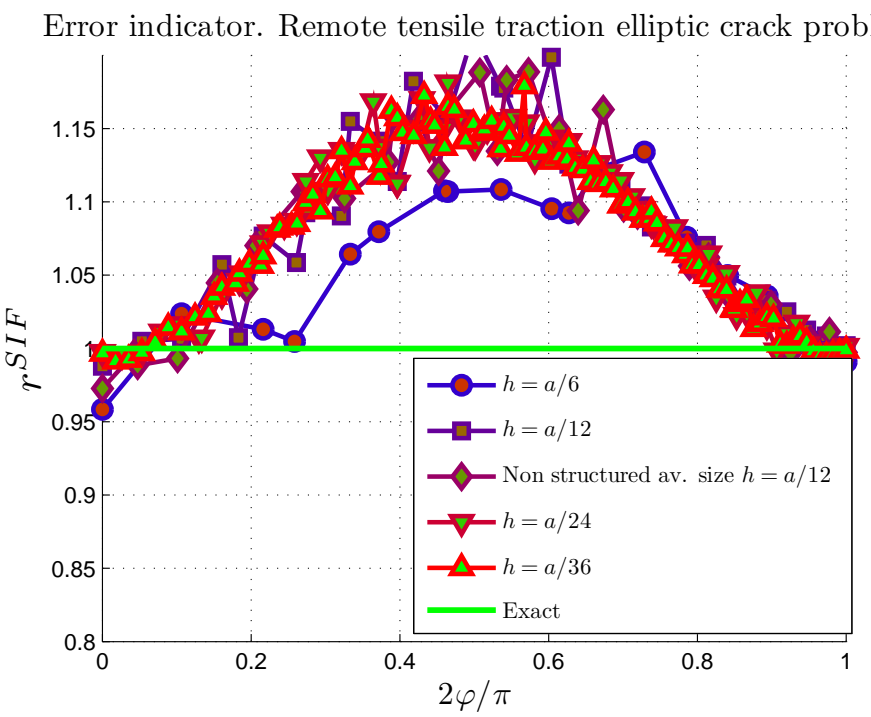

Fig. 37. Error indicator distribution results for the mesh sequence along the crack front for the three-dimensional elliptic crack under remote tensile traction, including the non structured mesh. 


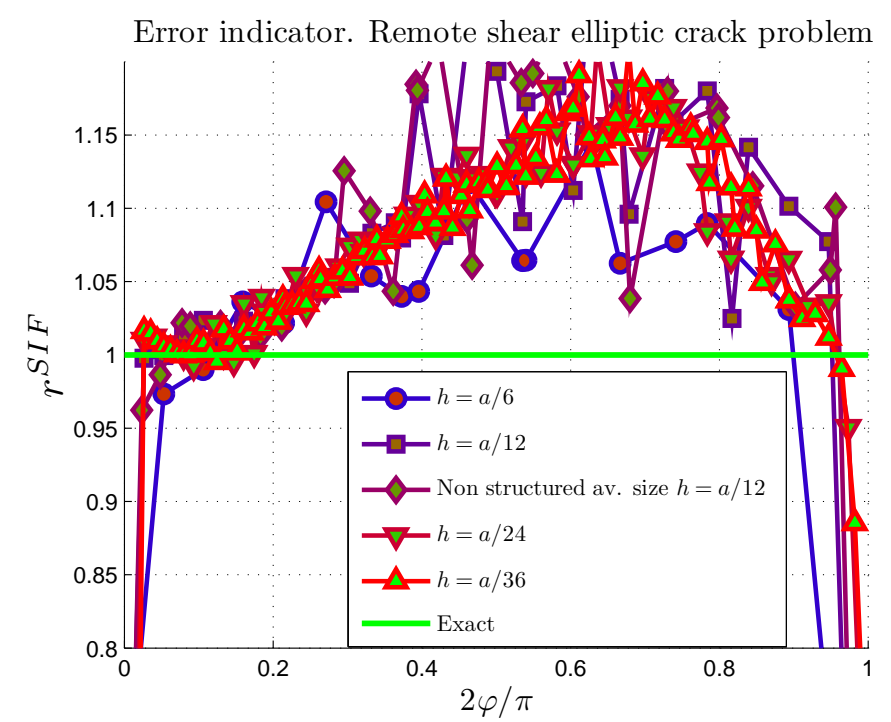

Fig. 38. Error indicator distribution results for the mesh sequence along the crack front for the three-dimensional elliptic crack under remote shear. 


\section{Conclusions}

In the present work, we have introduced some contributions to the domain integral formulation for curved cracks in 3-D. One of the contributions is based on the explicit formulation of the level sets basis using di erential geometry concepts. The interaction integral formulation includes also minor changes to minimize the effect of the auxiliary fields. We also remark the domain extrac-tion dependency with relative topology of crack and mesh and its effect. We have also proposed a curvilinear derivation of correction terms, along with its numerical implementation, and the introduction of an error indicator benefit-ing from the high convergence rate of the $J$-Integral.

A curvilinear correction to the $J$-integral and the interaction integral expression for XFEM for analysis of curved and non-planar cracks has been introduced. The proposal is sufficiently general to be used in other domain integrals in applications where curvilinear magnitudes have to be considered. The correction is dependent on the level of refinement, as it imposes limits in the level set information of the crack description and the information is stored at the nodes and interpolated within the elements. The correction allows to capture the behavior of SIFs of curved and non-planar cracks as in $[9,10]$, but it does not rely of the analytical information of the crack geometry as it depends only on the level set curvilinear coordinates. Therefore the correction is clearly an improvement of the previous interaction integrals proposed in the litterature. The results, based on numerical examples, show that the correction is needed to improve the convergence to the exact value in non-planar-cracks and introduces an improvement for curved cracks. The amount of this improvement depends on the local curvature, crack description and the mesh element size.

A SIF error indicator is also introduced. The proposed error indicator relies on the existing relationships between SIFs and the different domain integrals: $J$-integral and interaction integral. The relationships allow to improve the interaction integral results if the error in these results is not large.

\section{ACKNOWLEDGEMENTS}

This work has been carried out within the framework of the research projects DPI2007-66995-C03-02 and DPI2010-20990 financed by the Ministerio de Economía y Competitividad. The support of the Generalitat Valenciana, Programme PROMETEO 2012/023 is also acknowledged. 


\section{References}

[1] Moës N, Dolbow J, Belytschko T. A finite element method for crack growth without remeshing. Int J Numer Methods Engng 1999;46:131-150.

[2] Sukumar N, Moës N, Moran N, Belytschko T. Extended finite element method for three-dimensional crack modelling. Int $J$ Numer Methods Engng 2000;48:1549-1570.

[3] Rannou J, Gravouil A, and Baetto-Dubourg MC. A local multigrid X-FEM strategy for 3-D crack propagation. Int J Numer Methods Engng 2009;77:581600

[4] Sethian JA. Level set methods; evolving interfaces in geometry, fluid mechanics, Computer Vision and Material Sciences, Cambridge University Press, 1996.

[5] Osher S, Fedkiw RP. Level set methods: An overview and some recent results. J Comput Phys 2001;169:463-502.

[6] Moës N, Gravouil A, Belytschko T. Non-planar 3D crack growth by the extended finite element and level sets - Part I: Mechanical model. Int $J$ Numer Methods Engng 2002;53:2549-2568.

[7] Gravouil A, Moës N, Belytschko T. Non-planar 3D crack growth by the extended finite element and level sets - Part II: Level set update. Int $J$ Numer Methods Engng 2002;53:2569-2586.

[8] Duflot M. A study of the representation of cracks with level sets. Int J Numer Methods Engng 2006;70(11):1261-1302.

[9] Gosz M, Dolbow J, Moran B. Domain integral formulation for stress intensity factor computation along curved three-dimensional interface cracks. Int $J$ Solids Struct 1998;35(15):1763-1783.

[10] Gosz M, Moran B. An interaction energy integral method for computation of mixed-mode stress intensity factors along non-planar crack fronts in three dimensions. Engng Fracture Mech 2002;69:299-319.

[11] Walters MC, Paulino GH, Dodds RH Jr. Interaction integral procedure for 3-D curved cracks including surface traction. Engng Fracture Mech 2005;772:16351663.

[12] Nahta R, Moran B. Domain integrals for axisymmetric interface crack problems. Int J Solids Struct 1993;30(15):2027-2040.

[13] González-Albuixech VF, Giner E, Tarancón JE, Fuenmayor FJ, Gravouil A. Convergence of domain integrals for stress intensity factor extraction in 2-D curved cracks problems with the extended finite element method. Int $J$ Numer Methods Engng 2013; DOI: 10.1002/nme.4478. In press.

[14] Huang R, Sukumar N, Prévost J-H. Modeling quasi-static crack growth with the extended finite element method. Part II: Numerical applications. Int $J$ Solids Struct 2003;40(26):7539-7552. 
[15] Sukumar N. Element partitioning code in 2-D and 3-D for the extended finite element method. http://dilbert.engr.ucdavis.edu/ suku/xfem, 2000.

[16] Park K, Pereira JP, Duarte CA, Paulino GH. Integration of singular enrichment functions in the generalized/extended finite element method for three-dimensional problems. Int J Numer Methods Engng 2009; 78:1220-1257.

[17] Rice JR. A path independent integral and the approximate analysis of strain concentration by notches and cracks. J Appl Mech 1968;35:379-386.

[18] Shih CF, Moran B, Nakamura T. Energy release rate along a three-dimensional crack front in a thermally stressed body. Int J Fract 1986;30:79-102.

[19] Eshelby JD. The elastic energy-momentum tensor. J Elasticity 1975;5(3-4):321335 .

[20] Moran B, Shih CF. Crack tip and associated domain integrals from momentum and energy balance. Engng Fracture Mech 1987; 27(6):615-642.

[21] Murakami Y. Stress Intensity Factors Handbook, Pergamon Press 1987.

[22] Rajaram H, Socrate S, Parks DM. Application of domain integral methods using tetrahedral elements to the determination of stress intensity factors. Engng Fracture Mech 2000;66:455-482.

[23] Eriksson K. A general expression for an area integral of a point-wise $J$ for a curved crack front. Int $J$ Fract 2000;106(19):65-80.

[24] Nakamura T, Parks DM. Three-dimensional stress field near the crack front of a thin elastic plate. J Appl Mech 55 1988;55:805-813.

[25] Muskhelishvili NI. Some basic problems in the mathematical theory of elasticity. Leiden: Noordhoff; 1952.

[26] Irwin GR. Crack-extension force for a part-through crack in a plate. $J$ Appl Mech 1962;29(4):651-654.

[27] Kassir MK, Sih GC. Three dimensional stress distribution around an elliptical crack under arbitrary loadings. J Appl Mech 1966;33:601-611.

[28] Nuller B, Karapetian E, Kachanov M. On the stress intensity factor for the elliptical crack. Int J Fract 1998;92(2):15-20. 